\title{
Identification of New In Vivo TonB-FepA Rendezvous Sites
}

5 Kathleen Postle ${ }^{1,2^{*}}$, Kelvin Kho ${ }^{1,3}$, Michael Gresock ${ }^{1,4}$, Joydeep Ghosh ${ }^{2,5}$, and

6 Ray Larsen ${ }^{2,6}$

7

Running title: In vivo TonB-FepA interactions

Keywords: TonB, FepA, disulfide bond, amphipathic helix, energy

transduction

Department of Biochemistry and Molecular Biology, The Pennsylvania State

* Correspondence: ${ }^{1}$ Kathleen Postle, Department of Biochemistry and

17 16802, USA. e-mail: postle@psu.edu, Ph. 1-814-863-7568, Fx. 1-814-863-7024 
${ }^{2}$ School of Molecular Biosciences, Washington State University, Pullman, WA

20

99163

21

Current addresses:

22

${ }^{3}$ Institut Pasteur, Unité Biologie et Génétique de la Paroi Bactérienne,

23

Département de Microbiologie, Paris 75015, France

24

${ }^{4}$ Department of Biology, University of Mt. Union, Alliance OH, 44601

25

${ }^{5}$ Division of Cellular and Gene Therapies, US FDA Center for Biologics

26

Evaluation and Research, Silver Spring, MD

27

${ }^{5}$ Department of Biological Sciences, Bowling Green State University, Bowling

28

Green OH, 43403

29

30

31 
32

33

34

35

36

37

38

39

40

41

42

43

44

45

46

47

48

49

50 sites of interaction in vivo. The TonB Cys substitutions in the core of an essential

51 carboxy terminal amphipathic helix (residues 199-216) were compared to TonB

\section{ABSTRACT}

The TonB system of Gram-negative bacteria uses the protonmotive force of the cytoplasmic membrane to energize active transport of large or scarce nutrients across the outer membrane by means of customized beta-barrels known as TonBdependent transporters (TBDTs). The lumen of each TBDT is occluded by an amino-terminal domain, called the cork, which must be displaced for transport of nutrients or translocation of the large protein toxins that parasitize the system. A complex of cytoplasmic membrane proteins consisting of TonB, ExbB and ExbD harnesses the protonmotive force that TonB transmits to the TBDT. The specifics of this energy transformation are a source of continuing interest. The amino terminal domain of a TBDT contains a region called the TonB box, that is essential for the reception of energy from TonB. This domain is the only identified site of $i n$ vivo interaction between the TBDT and TonB, occurring through a non-essential region centered on TonB residue Q160. Because TonB binds to TBDTs whether or not it is active or even intact, the mechanism and extent of cork movement in vivo has been challenging to discover. In this study, we used in vivo disulfide crosslinking between eight engineered Cys residues in Escherichia coli TonB and 42 Cys substitutions in the TBDT FepA, including the TonB box, to identify novel 
52 Q160C interactions. Functionality of the in vivo interactions was established when

53 the presence of the inactive TonB H20A mutation inhibited them. A previously

54 unknown functional interaction between the hydrophilic face of the amphipathic

55 helix and the FepA TonB box was identified. Interaction of Q160C with the FepA

56 TonB box appeared to be less functionally important. The two different parts of

57 TonB also differed in their interactions with the FepA cork and barrel turns. While

58 the TonB amphipathic helix Cys residues interacted only with Cys residues on the

59 periplasmic face of the FepA cork, TonB Q160C interacted with buried Cys

60 substitutions within the FepA cork, the first such interactions seen with any TBDT.

61 Both sets of interactions required active TonB. Taken together, these data suggest a

62 model where the amphipathic helix binds to the TonB box, causing the

63 mechanically weak domain of the FepA cork to dip sufficiently into the

64 periplasmic space for interaction with the TonB Q160 region, which is an

65 interaction that does not occur if the TonB box is deleted. The TonB amphipathic

66 helix also interacted with periplasmic turns between FepA $\beta$-strands in vivo

67 supporting a surveillance mechanism where TonB searched for TBDTs on the

68 periplasmic face of the outer membrane. 


\section{INTRODUCTION}

71 The TonB system of Escherichia coli appears to be an answer to the challenges

72 posed to Gram-negative bacteria by their dual membrane cell envelope. In

73 particular, the outer membrane is a largely protective sieve with a diffusion cut-off

74 of around $600 \mathrm{Da}$ in E. coli (1). To capture large, scarce, essential nutrients, the

75

76

77

78

79

80

81

82

83

84

85

86

87

88

89 outer membrane displays high-affinity customized $\beta$-barrels for active transport of diverse ligands (2). The energy for the active transport across the essentially unenergized outer membrane comes from a complex of cytoplasmic membrane proteins, TonB, ExbB, and ExbD. This complex harvests the cytoplasmic membrane proton gradient and transforms it into mechanical energy which drives vectoral transport of ligands through TBDTs and into the periplasmic space (3) (Fig. 1). Because these transporters bind directly only to TonB and not ExbB or ExbD during the energy transduction, they have been termed TonB-dependent transporters (TBDTs) (4). There is some confusion in the historical literature whereby they were first called TonB-dependent receptors because they were initially identified as receptors for colicins and bacteriophages, now known to be opportunistic agents (5-7). They have also been called TonB-gated transporters, TGTs, (8) and ligand-gated porins, LGPs, (9). Escherichia coli K12 encodes nine different TBDTs mostly devoted to acquisition of iron by various means with one devoted to cobalamin transport. While E. coli has dedicated TBDTs for a variety 
90 of siderophores, enterochelin (a.k.a. enterobactin) is the single iron-chelating

91 siderophore that E. coli synthesizes and excretes to capture iron from its

92 environment [for a review see (10)]. The TBDT that provides for the recovery of

93 iron-bearing enterochelin is FepA.

94

95

96

97

98

99

100

101

102

103

104

105

106

107

108

Each TBDT consists of a 22 -stranded $\beta$-barrel, the lumen of which is

occluded by an essential internal globular domain of $\sim 150$ residues called the cork (or hatch) [(11); for a review see (2)]. Because they are similar in structure, the results from one TBDT largely apply to most TBDTs. The mechanism by which

TBDTs actively transport ligands such as the iron-siderophore enterochelin or cobalamin across the outer membrane remains a mystery, but there is general agreement that the cork must somehow move.

A TBDT cork has both a mechanically weak (approximately residues 1-70) and mechanically recalcitrant domain (approximately residues 70-150) both in vivo and in vitro $(9,12-14)$. An essential motif of five to seven mostly conserved residues known as the TonB box occupies the amino terminus of the mechanically weak domain. The TonB boxes of TBDTs are interchangeable, indicating that they do not mediate ligand specificity $(15,16)$.

The precise energy-transducing interaction of TonB with TBDTs has been challenging to define by structural determinations in vitro because ExbB and ExbD 
109 functions, and the protonmotive force of the cytoplasmic membrane are all

110 required for TonB-dependent energy transduction. Furthermore, in vitro and in

111 vivo TonB binds to TBDTs regardless of its ability to transduce energy, (17-22),

112 suggesting that certain residue-specific interactions result in energy transduction

113 while other interactions fail to accomplish it.

114 The TonB protein is anchored in the cytoplasmic membrane by its

115 hydrophobic amino terminal signal anchor with the rest of the protein (residues 33-

$116239)$ occupying the periplasmic space [(23), Fig.1]. Interestingly, there are no

117 essential residues in TonB $(17,24-28)$. In fact, even the His20 residue in the

118 transmembrane domain can be replaced with non-protonatable Asn and retain full

119 function, suggesting that the H20A mutation used in that study renders TonB

120 inactive through steric distortion of its transmembrane domain in complex with

121 ExbB and ExbD transmembrane domains (28).

122

There are, however, seven residues in the periplasmic TonB carboxy

123 terminus (out of 90 sequentially scanned) that are functionally important (Y163,

124 F180, G186, F202, W213, Y215, and F230) (27). With the exception of G186,

125 these residues represent the complete set of aromatic residues in the last 90

126 residues of the carboxy terminus from $150-239$, with the only other aromatic

127 residue in the entire periplasmic domain (residues 33-239) being F125. We think

128 these seven residues are the key because: 
1) When substituted with Ala or Cys, each of these residues exhibits an

131 distinct for each of the seven substitutions $(27,29)$. They appear to be the means parasitize them (30). In contrast, Cys substitution at the only other aromatic residue, TonB F125C, supports wild-type activity. substitutions at any of the seven residues $(17,27,29)$. It therefore seems to set a maximal boundary on the active domain of the TonB carboxy terminus from G186 to F230, which contains a single amphipathic helix (residues 199-216). 
149 active domain of the carboxy terminus, forms triplet homodimers that, like wildtype TonB, still fractionate $\sim 40 \%$ with the outer membrane, indicating that the

151 subsequent, more carboxy-terminal residues, including especially the amphipathic

152 helix, are free to undergo necessary conformational changes (31).

TonB is the limiting protein in the TonB system (32) and different TBDTs

154 must compete for its attention (33). TonB therefore interacts transiently with

155 ligand-loaded TBDTs in $E$. coli K12, giving rise to an energy transduction cycle

(67). Over the years we have defined stages in that cycle in vivo, the model for

157 which is depicted in Fig. 2.

In the model, the TonB carboxy termini of homodimers are conformationally

159 dynamic while the amino termini remain stably homodimerized throughout the

160 energy transduction cycle (31). Protonmotive force of the cytoplasmic membrane

161 is transduced into active transport at the outer membrane through sequential

162 contacts by the TonB carboxy terminus, first with itself, then with the ExbD

163 carboxy terminus, then with a TBDT (31). In vivo interaction sites between TonB

164 homodimers and between TonB-ExbD heterodimers have been identified, and a

165 common region between them is the TonB amphipathic helix (residues 199-216;

166 Fig. 3) (27, 34, 35). The primary goal of this study was to determine if the TonB

167 amphipathic helix played a role in contact with FepA. 
169 the TonB box, the cork, and the $\beta$-strand turns of the barrel. A novel in vivo

170 interaction between Cys substitutions in the hydrophilic face of the essential TonB

171 amphipathic helix and Cys substitutions in the essential FepA TonB box was

172 identified. Interactions between TonB amphipathic helix and $\beta$-strand turns of the

173 FepA barrel were identified, providing the first in vivo support for a surveillance

174 model where TonB searches for a TBDT. The difference in interaction profiles

175 with the mechanically weak domain of the FepA cork between the TonB

176 amphipathic helix and TonB Q160 led to a model where the amphipathic helix

177 pulls the mechanically weak domain of the FepA cork out of the barrel sufficiently

178 that TonB Q160 interacts with otherwise buried residues. 
182

183

184

185

186

187

188

189

190

191

192

193

194

195

196

197

198

199

200

201

\section{RESULTS}

\section{The carboxy terminal TonB amphipathic helix is essential for TonB system}

\section{activity.}

The TonB region from $\sim$ R158-N162, centered on TonB Q160 interacts with

the TonB boxes of TBDTs in vivo [Fig. 4A; $(4,36)]$. While the TonB box as a

whole is essential for TBDT activity, its precise amino acid composition is tolerant

of substitutions except for structure-breaking residues such as L8P in BtuB or I14P

in FepA, substitutions that result in inactivation of the TBDT $(4,15,36,37)$. Even

then, the TonB Q160 region still interacts with the BtuB L8P mutant TonB box in

vivo, but in a different way (4).

Based on the position of residue Q160 within the TonB carboxy terminus, the solved crystal structures of TBDTs, and the solved crystal structures of TonB carboxy termini in complex with those TBDTs (Fig. 4B), it seems unlikely that Q160 contacts a TBDT without significant conformational changes to make the TonB box more accessible. The deletion of the proline rich domain that accounts for $\sim 100 \AA$ of TonB's reach to a TBDT has negligible effect upon its activity unless the periplasmic space is artificially expanded by transient exposure to high salt $(24,38,39)$. This observation suggests that residues nearer the carboxy 
202 terminus of TonB could be more important. There is also evidence that unknown

203 regions in addition to the FepA TonB box and TonB Q160 are involved in

204 transport $(12,40)$. Furthermore, the TonB Q160 region is not essential, suggesting

205 that its role in contacting a TBDT is not essential (11).

206

The periplasmic TonB carboxy terminus contains an amphipathic helix

207 (residues 199-216) which has been intriguing for many years $(24,29,41)$. It

208 includes three of the seven functional carboxy terminal residues, F202, W213 and

209 Y215. To further explore the role the amphipathic helix plays in the mechanism of

210 TonB-dependent energy transduction, we deleted the amphipathic helix codons

211 199-216 from plasmid pKP325, resulting in plasmid-encoded TonB $\triangle \mathrm{AH}$ (Fig. 5A).

212 Plasmids expressing chromosomal levels of TonB $\mathrm{BAH}_{\Delta \mathrm{H}}(\mathrm{pK} 476)$ were unable to

213 complement KP1477 $(\Delta t o n B)$ in cross-streaks against colicins B, Ia, and M, the

214 most sensitive assays known for TonB function, requiring $\sim 1$ active TonB

215 molecule per cell (42).

216

$\mathrm{TonB}_{\triangle \mathrm{AH}}$ fractionated on sucrose density gradients with $\sim 60 \%$ located in the

217 cytoplasmic membrane fractions and $\sim 40 \%$ with the outer membrane fractions

218 (Fig. 6A), the same proportions with which wild-type TonB fractionates (43). It is

219 not known what causes TonB to bind sufficiently tightly to outer membrane

220 components that $\sim 40 \%$ is pulled out of its complex with cytoplasmic membrane

221 proteins $\mathrm{ExbB}$ and $\mathrm{ExbD}$ to fractionate with the outer membrane. One hypothesis 
222 is that TonB outer membrane fractionation reflects a transient tight association

223 with outer membrane molecules--likely TBDTs--during Stage IV in the energy

224 transduction cycle (Fig. 2). While the region required for outer membrane

225 fractionation, residues 175-239, includes the amphipathic helix (43) and is

226 responsible for the ability of TonB to formaldehyde crosslink to FepA (37), this

227 result indicated that the amphipathic helix was not the region responsible for

228 fractionation of TonB with the outer membrane.

230 Alternatively, it could have been due to the simultaneous deletion of residues

231 F202, W213, and Y215, since combination of two Ala substitutions at any of those

232 residues renders TonB inactive $(27,29)$. Individual Cys substitutions from E203

233 through R212 have little phenotypic effect (27). To retain F202, W213, and Y215

234 and broadly restore the overall structure, we shifted the 10-amino acid core region

235 within the helix out of frame starting at residue 203 and shifted it back into frame

236 at residue $213\left(\mathrm{TonB}_{\mathrm{fs}}, \mathrm{pKP} 372\right)$. The result for TonB $\mathrm{fs}$ was that the predicted

237 helical region was shortened slightly to encompass residues 201-209 (as analyzed

238 by JPRED), lost much of its amphipathic character, and at residue 210, Met was

239 substituted with Cys due to the frameshift (Fig. 5). Like TonB $\triangle \mathrm{AH}$, TonB $_{\mathrm{fs}}$ was

240 completely insensitive in the colicin assays. 
242 homodimers. Like those of the Cys substitutions in functionally important

243 residues, the $\mathrm{TonB}_{\mathrm{fs}}$ triplet homodimers fractionated essentially entirely with the

244 cytoplasmic membrane, suggesting that they had trapped TonB at a stage in the

245 energy transduction cycle before TonB associates with the outer membrane [Fig.

$2466 \mathrm{~B} ;(17,27)]$. TonB M210C in the context of otherwise wild-type residues is

247 active and does not form triplet homodimers (27).

251 remained monomeric and was also found in the outer membrane on longer

252 exposures (data not shown), its inactivity was not due to $100 \%$ entrapment as a

253 triplet homodimer (Fig. 5B). We concluded that one or more aspects of the core amphipathic helix domain were required for TonB activity. 
261 disulfide crosslinking of TonB and FepA Cys substitutions expressed at

262 chromosomal levels, followed by electrophoresis on non-reducing SDS gels and 263 immunoblotting with anti-TonB monoclonal antibodies. We surveyed crosslinking by TonB amphipathic helix residues R204C, 265 V206C, N208C, A209C, and R212C (Fig. 5). When expressed at chromosomal 266 levels, each of the TonB Cys substitutions was at least $60 \%$ active in ${ }^{55} \mathrm{Fe}$ -

267 ferrichrome transport assays (Table 1). TonB Q160C, over 100\% active and a 268 known site of interaction with other TBDT TonB boxes, was also tested for 269 comparison to the amphipathic helix Cys substitutions [Table $1 ;(4,16)]$. The 270 FepA TonB box Cys substitutions tested were D12C, T13C, I14C, V15C, V16C 271 and T17C; FepA T13C is fully active (44) as was FepA V16C (Table 1) with the 272 rest assumed to be active. The amphipathic helix substitutions at R204C, N208C and R212C formed

274 disulfide-linked complexes with all FepA TonB box Cys residues except N208C 275 with FepA T17C (for unknown reasons) (Fig. 7A). In contrast, TonB V206C and 276 A209C did not form significant complexes with any of the FepA TonB box Cys 277 residues, thus constituting a non-reactive hydrophobic face of the amphipathic 278 helix. Steady state levels of monomeric TonB from samples in Fig. 7A are 279 presented in Fig. 7B. 
281 relevant, each TonB Cys substitution was also paired with the H20A mutation in TonB through its carboxy terminus, a step necessary for formation of carboxy terminal TonB-ExbD heterodimers and the subsequent correct interaction of the

285 TonB carboxy terminus with FepA [Figs. 2 and 4A, $(18,31)]$. TonB H20A

286 epitomizes the behavior of inactive TonB because it still interacts at unknown sites 287 with FepA in vivo $(17,18)$. The TonB H20A mutation rendered all the TonB Cys substitutions inactive (Table 1). N208C and R212C, indicating that the H20 wild-type versions were engaging in biologically relevant interactions. The possibility that H20A somehow promoted new interactions with the hydrophobic face (substitutions V206C and A209C) was excluded since no complexes were observed. These results indicated that the TonB its role in TBDT reception of TonB-transmitted energy. The TonB amphipathic

296 helix is the first known alternative to the TonB Q160 region for contact with the 297 FepA TonB box. In the solved co-crystal structures of the TonB carboxy terminus with the

299 TBDTs BtuB and FhuA, TonB residues R204, N208 and R212 of the amphipathic 
300 helix interact with the barrels, but not the TonB boxes [Fig. 8, $(20,45)]$. The lack

301 of interaction with the TonB amphipathic helix in those elegant co-crystal

302 structures supports the idea that the TonB carboxy terminus remains able to bind to

303 TBDTs and other proteins in an "un-energized" conformation $(17,18,46,47)$.

304

305

TonB $Q 160$ interaction with the FepA TonB box is only partially dependent on

306

307

308

309

310

311

312

313

314

315

316 317 relative levels of TonB-FepA complex formation.

\section{TonB activity.}

Although the interaction of the TonB Q160 region with the TonB boxes of several TBDTs has been well-documented both in vivo and in vitro $(4,15,16,48$ $51)$, there has not yet been an analysis of how TonB Q160 interacts with the TonB box of FepA nor an analysis of effects of inactive TonB upon any TBDT TonB box interaction. In Fig. 7A, TonB Q160C crosslinked with all of the FepA TonB box Cys substitutions, consistent with its behavior seen previously for BtuB Cys substitutions in the TonB box (4). FepA V16C was chosen as the standard in all subsequent experiments because it exhibited the highest degree of disulfide crosslinking to TonB Q160C (Fig. 7A, left panel, lane 3), allowing comparisons of 
All of the interactions gave rise to an apparent higher and an apparent lower in the TonB Cys substitutions studied here, all of which carry the C18G substitution that removes the single native Cys. Second because the complexes are also detected with anti-FepA polyclonal antibodies, ruling out participation of a different protein (data not shown). And third, because similar doublets are also 
338

339

340

341

342

343

344

345

346

347

348

349

350

351

352

353

354

355

356

357

Other FepA Cys substitutions assessed include the cork and periplasmic turns between $\beta$-strands of the barrel.

Interaction of the TonB Cys substitutions with several additional FepA Cys

substitutions other than the TonB box was assayed to identify potential additional

sites of interaction by both the TonB amphipathic helix and Q160. In the

mechanically weak domain of the FepA cork, L23, S29, T32, A33, D34, and E35

are periplasmically accessible in the crystal structure. Residues A42, S46, and G54

are buried; T51 is partially buried. L85 is in the mechanically recalcitrant domain

and is partially buried [Fig. 9]. FepA residue T32, semi-conserved across TBDTs

(53), and the less-well-conserved A33 were included because they bind to TonB at

unknown sites and respond differentially to the presence and absence of ligand in

FepA photocrosslinking studies in vivo (40). G54 exhibits a modest change in

periplasmic exposure upon ligand binding (9).

FepA residues in the mechanically recalcitrant segment of the FepA cork

(V91, S92, S112, E120, V124, A131, V142, I145) were also evaluated to detect

possible cork movements not observed previously [(12) Fig. 10]. Also evaluated

were FepA R75, R126, E511, and E567, which form part of the "lock region", with

R75 and R126 in the recalcitrant domain of the FepA cork, and E511 and E567

positioned in the barrel $(12,14,54)$. The lock region is proposed to be important 
358 for transport but not binding of ligand, with the positively and negatively charged

359 residues forming a structure that keeps the cork bound to the barrel $[(36,55,56)$.

360

Contact with residues in periplasmic turns between $\beta$-strands of the FepA

361

barrel, including the cork and barrel linker (D185, P243, D298, D356, D422,

362 D455, D519, E576, D618, and D664; Fig. 11) were assessed, something that has

363 not been investigated before for any TBDT. FepA residue E152, which marks the

364 transition from cork to barrel and residue T722, the third residue from the carboxy

365 terminus of FepA, were also included in the analysis.

366

367

to form disulfide crosslinks with TonB Cys substitutions.

368

369

370

TonB amphipathic helix interactions extend into the mechanically weak region

of the FepA cork.

371

372

To define the boundaries of reactive residues in the TonB amphipathic helix,

373 the set of core TonB amphipathic helix Cys substitutions was expanded to include

374 M201C and R214C (Fig. 5). Each TonB Cys substitution was assayed pairwise at

375 least twice in combination with the 35 additional FepA Cys substitutions outside

376 the TonB box. 
TonB M201C and R214C gave little to no interaction with any FepA Cys, periplasmically-accessible FepA cork Cys residues (L23C, S29C, T32C, A33C, and D34C, but not E35C), with S29C and A33C interactions being the most abundant (Fig. 13A). 
FepA S29 is located close to the center of the first $\beta$-strand of the cork. It is within

\section{The TonB amphipathic helix interacts with periplasmic FepA $\beta$-strand barrel}

407

408

409

410

turns.

In vivo, TonB interacts with FepA at one or more sites before it interacts with the FepA TonB box (12). Instead of FepA S29, the periplasmic $\beta$-strand turns of TBDTs could constitute such sites.

The last 150 residues of the TonB periplasmic domain, within which the amphipathic helix resides, have a calculated $\mathrm{pI}$ of 10.4 (41). It seemed logical that this TonB domain would be most likely to interact with the negatively charged residues of FepA barrel turns, where there appears to be one in nearly every turn 
416 (except Pro243 in turn 2), including Glu152 in the linker region between cork and

$417 \quad \beta$-strand 1. Such interactions would likely be mediated through additional

418 residues of TonB and FepA that would bring the now Cys-substituted residues into

419 proximity (Fig. 11).

420

All of the FepA Cys substitutions in $\beta$-strand turns exhibited the ability to

421 support fully wild-type levels of ${ }^{55}$ Fe-enterochelin transport except FepA E152C

422 and T722C, where the levels dropped to $\sim 60 \%$ (Table 1 ). Two Cys substitutions

423 in the lock region of the barrel, E511C and E567C, were also assayed. FepA

424 E511C had little effect on FepA activity, and E567C reduced activity to 65\% of

425 wild-type. Cys substitutions in the cork components of the lock region, R75C and

426 R126C, reduced transport to $\sim 40 \%$ of wild-type levels but did not eliminate it.

427 Thus, neither the charged residues in the periplasmic $\beta$-strand turns nor the lock

428 region residues were individually essential for FepA function.

Several sites of interaction between the TonB amphipathic helix residues

431 summarized in Fig. 12C.

433 also seen with the FepA cork Cys substitutions (Fig. 13B). In contrast to

434 interactions with the FepA cork, the apparent masses of the complexes with 
436 in the case of the latter three for unknown reasons. In contrast to interactions with

437 the cork, the $\beta$-strand barrel complexes were largely insensitive to the presence of

438 TonB H20A. Overall intensities of the complexes remained similar with both

439 TonB H20 and TonB H20A versions of R204C, but in the case of TonB H20A, the

440 complexes appeared to slightly shift their abundance to the higher mass complex-

441 again for unknown reasons.

442 For TonB N208C, weak interactions were detected with FepA E152C,

443 P243C, D298C, and D519C, each of which was H20-dependent (Fig. 14A). In

444 addition, TonB N208C made a very high level of contacts with D422C whether

445 TonB H20 or H20A was present. TonB R212C made a moderately high level of

446 H20-sensitive contacts with P243C, D422C, and D519C, whereas the contact with

447 D455C was largely H20-insensitive (Fig. 14B). Thus, both functional and non-

448 functional TonB amphipathic helix contacts occurred with the FepA $\beta$-strand turns.

449

450 TonB Q160 interactions include buried cork residues.

451

452

Previous studies of TonB Q160C with TBDTs have been confined to the

453 TonB box. Since the TonB amphipathic helix made FepA contacts outside the

454 TonB box, we wanted to explore the possibility that Q160 did so as well. 
S46C and T51C, with the highest degree of interactions occurring with D34C and

S42C. The presence of the H20A mutation greatly diminished, and in most cases

461

eliminated, the crosslinking detected, suggesting that when TonB was inactive, the

462

ability of Q160C to make contacts within the mechanically weak domain of the

463

FepA cork was entirely prevented, unlike the interaction with the FepA TonB box

464

(Fig. 7A; 15C)

465

The disulfide crosslinking between TonB Q160C and FepA A42C or S46C

466

was notable because A42 and S46 are buried in the FepA crystal structure. TonB

467

Q160C crosslinks with FepA A42C were as abundant as those between the

468

standard TonB Q160C-FepAV16C pair (Figs. 9 and 15C). FepA A42 and S46 are

469

positioned in a plane approximately mid-way between top and bottom of the cork.

470

FepA A42 is on the interface with the FepA barrel. FepA S46 is entirely buried

471 within the cork (Fig. 9). These key observations are incorporated into a model in

472 the discussion. 
475 weak H20-specific interactions with FepA G54C were also observed on long

476

477

478

479

480

481

482

483

484

485

486

487

488

489

490

491

492

493

494

exposures (Fig. 16, lane 5). Ma et al. previously observed modest periplasmic

exposure of FepA G54C in the presence of enterochelin (9). With the exception of

scarcely detectable interactions with D519C in a barrel turn, no interactions of

TonB Q160C with any of the remaining FepA Cys substitutions from V91C

through I145C (the mechanically recalcitrant domain), the lock region or the barrel

turns were detected no matter how long the exposures were (Figs. 12B and 12C;

data not shown). These observations form an important part of the model

presented in the discussion.

\section{Deletion of the FepA TonB box prevents Q160C FepA cork interactions.}

Prior to the experiments above, the interaction of TonB Q160 with TBDT sites other than their TonB boxes had not been tested. Since several additional interactions were identified, we attempted to determine an order of events by analyzing the effect of a FepA TonB box deletion on complex formation with the FepA Cys substitution D34C with which TonB Q160C interacts with as abundantly as it does the FepA TonB box, and G54C, which is buried and where the interaction is weaker. In both cases, deletion of the FepA TonB box (residues 12-
17) essentially prevented the interaction (Fig. 16A, lanes 4 and 6). This finding 
495

496

497

498

499

500

501

502

503

504

505

506

507

508

509

510

511

512

513

514

suggested that prior contact by unspecified TonB residues with the FepA TonB

box was required for TonB Q160C to interact with FepA cork residues beyond the TonB box.

\section{TonB F202A, W213A lacks ExbD contact and inhibits the interaction of TonB}

\section{Q160C with the FepA TonB box.}

In TonB, the F202A and W213A mutations boundary the core of the amphipathic helix and, in combination, completely inactivate it (29). TonB F202A, W213A was used as a tool to better understand parameters of the interaction between TonB Q160C and FepA V16C in the TonB box.

At the time when we discovered the inactivity of TonB F202A, W213A, the TonB-ExbD interaction captured by in vivo formaldehyde crosslinking of TonB had yet to be identified, however we knew that such double Ala mutations in the carboxy terminus did not prevent formation of the disulfide-linked TonB triplet homodimers $(17,29)$. In this study, the effect of the F202A, W213A mutation as well as another double Ala mutation--F202A, Y215A--was to prevent formation of the Stage III TonB-ExbD formaldehyde crosslinked heterodimer $(18,31)$. It was particularly telling that even when the TonB double Ala mutants were overexpressed, there was still no formation of the TonB-ExbD heterodimer, which 
515 appears to play a key role in configuring TonB for successful energy transduction

516 to FepA (Fig. 17; Fig. 2, Stage III TonB-ExbD heterodimers).

517 When F202 and W213 are mutated individually, they support intermediate and assay-specific levels of TonB activity (29). Consistent with that, the TonB Q160C, F202A substitution was still able to form the Q160C-V16C complex (Fig. lane 4). Although the presence of the F202A, W213A double mutations rendered

521 TonB proteolytically unstable (Fig. 18, lane 5), as seen previously (29), it was

522 possible to increase the exposure time of the immunoblot to the point where the 523 level of monomer (Fig. 18, lane 6) was slightly greater than the level seen for the 524 TonB Q160C and its F202A derivative (Fig. 18, lanes 3 and 4). In the longer 525 exposure, it was clear that the ability of the TonB Q160C, F202A, W213A to form 526 complexes with FepA V16C was significantly diverted away from the FepA TonB.

527 Instead, the absence of a functional TonB carboxy terminus led Q160C to form

528 three complexes, too small to be complexes with FepA. The location and spacing 529 of the complexes were reminiscent of disulfide-linked TonB triplet homodimers 530 that represent three different conformations of the TonB carboxy terminus in vivo 531 (17).

\section{DISCUSSION}



membrane protonmotive force energy required for the active transport of ligands sole siderophore synthesized and secreted by E. coli (12). TonB sites focused on

546 TonB Q160 and the TonB carboxy terminal amphipathic helix and their potential

547 interactions with sites in the FepA TonB box, in both mechanically weak and

548 mechanically recalcitrant domains of the FepA cork, and in FepA $\beta$-strand barrel

551 membrane are all present (57). 
557 demonstrating that, although TonB Q160 has been the only site established to

558 interact with TBDTs, it is not sufficient for activity, and indicating that some

559 aspect of the last 65 TonB residues is essential (37). Within those last 65 residues,

560 we focused on the role of a sole amphipathic helix in the TonB carboxy terminus

561 (residues 199-216).

562

We found that the TonB amphipathic helix was essential either by deleting it

563 or by frameshifting it to maintain overall residue continuity. While this confirmed

564 the importance of the region, those results could also reflect an inability to form

565 TonB-TonB homodimers or TonB-ExbD heterodimers, both of which are

566 important for the energy transduction cycle $(27,31,34,35)$. We therefore asked if

567 the TonB amphipathic helix interacted directly with the only known essential

568 region of the TBDT, FepA, its TonB box.

569

Based on the in vivo disulfide crosslinking experiments, amphipathic helix

570 residues $\mathrm{R} 204 \mathrm{C}$, N208C and R212C defined a hydrophilic face that interacted with

571 Cys substitutions throughout the FepA TonB box, whereas the two residues on the

572 hydrophobic face, V206C and A209C did not interact with FepA. TonB R204C

573 was previously shown to be solvent exposed at some point in the energy

574 transduction cycle, consistent with localization on the hydrophilic face (58). 
575 Because these in vivo interactions were prevented by the presence of the

576 inactivating TonB H20A mutation, they comprised a set of novel, functional, and

577 specific interactions that have been identified between TonB and a TBDT for the

578 first time.

The amphipathic helix interaction with the TonB box is absent from solved

580

581

582

583

584

585

586

587

588

589

590

591

592

593

594

co-crystal structures of the TonB carboxy terminus ( residues $152-235)$ with

TBDTs BtuB and FhuA. The sidechains of the hydrophilic face residues R204C, $\mathrm{N} 208 \mathrm{C}$, and R212C are oriented towards the TBDTs and distant from the TonB boxes in both structures $(20,45)$, demonstrating either the difference between in vivo "energized" and inactive TonB, or differences between TBDTs.

Now that an essential TonB component has been identified that interacts

with an essential FepA component (TonB box), it is tempting to speculate that the

TonB amphipathic helix holds the entire key to the TonB energy transduction cycle

for $E$. coli. As a result of this study and previous work, residues within the TonB

amphipathic helix have now been recognized to participate in sequential

interactions with three different proteins_-TonB with itself, with ExbD and, here,

with FepA [(24, 59); Fig.2]. The amphipathic helix sequences are 55\% conserved

$(72 \%$ if $\mathrm{E} / \mathrm{D}, \mathrm{R} / \mathrm{K}$, and $\mathrm{W} / \mathrm{F}$ substitutions are considered equivalent) amongst

enteric bacteria, but barely conserved with Pseudomonas putida tonB, which does

not complement an $E$. coli tonB mutation $(24,59)$. 
596 vivo, but does not transmit energy to it $(18,28,44)$. Formation of disulfide

599 disulfide-linked complexes for some pairs likely represented lack of interactions, it 600 could also be that the interactions could not be trapped due to due to misorientation 601 of the thiol side chains or because the interactions were too transient. It is also 602 likely that other important in vivo regions of interactions remain to be discovered.

\section{A model: does the FepA cork "fish" for the TonB Q160 region?}

611 eliminating TonB activity [Fig. 4; $(24,39)$ ]. In addition, the amphipathic helix is

612 essential, whereas TonB Q160 and the region encompassing it (residues R158-

613 Q162) can be deleted without inactivating TonB (26). Why this region has been a 
614 source of second site suppressors for inactive TBDT TonB box mutations remains

615 a mystery $(48,49)$.

616

Because it is not essential, the wide range of in vivo contacts made by TonB

618 for other $E$. coli TBDTs but also, for the first time, residues throughout the region

619 of the mechanically weak domain of the FepA cork. Most importantly, two of the

620 interacting residues (A42, and S46) are buried within in the FepA cork (60). In

621 contrast to the partially H20-senstive Q160C interactions with the FepA TonB box,

622 both of these contacts were completely prevented if the TonB Q160C also carried

623 the inactivating $\mathrm{H} 20 \mathrm{~A}$ mutation, indicating that they fully represented the action of

624 functional TonB. The FepA region of TonB Q160C interaction ended just prior to

625 the beginning of the mechanically recalcitrant domain of the FepA cork.

626

TonB Q160C interacted as abundantly with FepA A42C as it did with the

627 FepA V16C standard within the TonB box, which was the most abundant

628 interaction seen in this entire study. Because FepA A42C is buried, this strongly

629 suggested that enough of the cork domain entered the periplasm to expose A42C,

630 along with S46C, to interaction with TonB Q160C. Our previous discovery of 20-

631 to-25-fold increases in periplasmic accessibility of the buried cork residues A42C,

632 S46C (and the partially buried T51C) in response to the addition of colicin B

633 ligand in vivo validates the finding here that at some point in the energy 
634 transduction cycle, these residues become available for interaction with TonB

$635 \mathrm{Q} 160 \mathrm{C}$ and likely neighboring residues $(4,12)$. In striking contrast, none of the 636 amphipathic helix Cys substitutions interacted with FepA Cys substitutions that 637 were buried within the cork, with targets limited to periplasmically accessible Cys 638 residues. The overall non-reactivity of the mechanically recalcitrant domain of FepA 640 in this in vivo study also validated our earlier finding of its resistance to being 641 periplasmically labeled in the presence of a large $(\sim 55 \mathrm{kDa})$ ligand, colicin $\mathrm{B}$. In 642 that study, two distinct possibilities were proposed for the mechanically 643 recalcitrant domain: first that it did not move and second that it moved but was 644 blocked from being labeled by the presence of another protein (12). Our studies 645 here did not exclude either possibility. For the first possibility to be true, colicin B 646 would need to denature on its way through a small opening, which, given its size 647 and structure as a dumbbell that fills the FepA lumen, seems unlikely (61).

648 Nonetheless, such denaturation has been observed for the amino-terminal domain 649 of colicin pyoS2 of $P$. aeruginosa through its TBDT FpvA in vivo (62).

650 Furthermore, the significantly smaller $(\sim 29 \mathrm{kDa})$ colicin $\mathrm{M}$, which parasitizes the

651 TBDT FhuA, uses a chaperone to fold it in the periplasm where it inhibits

652 peptidoglycan formation (63). A mechanically recalcitrant domain for the TBDT

653 BtuB has been characterized in vitro (14). 

and incidental to the action of the TonB amphipathic helix at the FepA TonB box.

671 with FepA TonB box residue V16C. As such, the double TonB mutation

672 somewhat mimicked the effect of the FepA TonB box deletion that prevented

673 Q160C interactions with FepA D34C and G54C, suggesting that TonB F202A, 
674 W213A might have inhibited the amphipathic helix from engaging the FepA TonB

675 box, preventing the FepA cork from fishing for TonB Q160C.

676 Consistent with our results revealing movement of the FepA cork, Majumdar

677 et al. engineered intra-cork disulfide bonds in FepA, most of which significantly

678 decreased Fe-enterochelin transport. The transport was restored in the presence of

679 reducing agent, indicating that there are required conformational changes within

680 the cork itself (52).

681

682

Comparison to results from in vivo photocrosslinking to study FepA dynamics

683

684

We previously identified FepA residues that interact with TonB using in vivo

685

photocrosslinking by the reagent $\mathrm{pBpa}$ inserted at sites of engineered amber

686

substitutions in fepA (40). A potential conformational switch signaling to TonB

687

that ligand (enterochelin) is bound was identified. FepA T32pBpa bound TonB in

688

the absence enterochelin whereas FepA A33pBpa bound TonB in its presence. In

689

the current study, both FepA T32C and A33C interactions occurred with both

690

TonB R204C in the amphipathic helix and Q160C without discrimination, perhaps

691 reflecting an average of ligand-bound and unbound states for FepA. FepA

$692 \mathrm{~S} 29 \mathrm{pBpa}, \mathrm{A} 42 \mathrm{CpBpa}, \mathrm{S} 46 \mathrm{pBpa}$, and T51pBpa in the mechanically weak domain

693 did not significantly photocrosslink to TonB, even though these variants all 
694 supported $\sim 75 \%$ activity. This could be because the interactions are too rapid to

695 capture, whereas in the current study, the disulfide bond formations would

696

697

698

699

700

701

702

703

704

705

706

707

708

709

710

711

712

713

potentially have been aided by the DsbA system (64).

Although the effect of a $d s b A$ mutation on disulfide formation in this study

was not tested, the effect on TonB triplet homodimer formation are informative

and suggest that the frustrating answer is: it depends. Plasmids expressing TonB

F125C, G186C, F202C, W213C, Y215C, or F230C substitutions were transformed into an isogenic $d s b A$ strain, KP1514 [W3110, $\Delta($ tonB, P14::blaM), dsbA::kan], and the degree of disulfide-linked triplet homodimer formation was compared to previous results from a $d s b A+\operatorname{strain}(27)$. TonB F125C, TonB Y215C and TonB

F230C showed greatly diminished triplet dimer formation in the absence of DsbA, with an intermediate decrease for W213C. In no case was triplet dimer formation entirely abolished. For TonB F202C and TonB G186C, there was no effect of the $d s b A$ mutation (Spicer and Postle, unpublished data).

In contrast to the present study, FepA E120pBpa and I145pBpa on the periplasmic face of the FepA cork, did photocrosslink to TonB (40). We do not have an explanation for these differences but note that the techniques are dissimilar, and the disulfide crosslinking studies here were congruent with our studies of in vivo FepA cork accessibility (12). 
Current in vivo approaches are not amenable to discovery of TonB aromatic

715

716

717

718

719

720

721

722

723

724

725

726

727

728

729

730

731

732

733

residue contacts with FepA.

This study revealed the importance of the TonB amphipathic helix core (residues 204-212) in contacting the TonB box of FepA. However, individual Cys substitutions within that core have no phenotype (27). Similarly, the sequences of TBDT TonB boxes contain little residue-specific information and indeed can be swapped for one another $(15,16)$. It is therefore unlikely that this set of interactions is responsible for the idiosyncratic phenotypes observed for Cys and Ala substitutions of the aromatic residues that boundary the amphipathic helix core-F202 and W213 among others.

We have been, unfortunately, thwarted in our ability to define the sites on FepA where TonB F202 and W213 interact due to two factors, both based on signal-to-noise ratios. First, Cys substitutions at these aromatic residues form a sufficiently high abundance of disulfide-linked triplet homodimers in vivo that any side reactions would be swamped out and difficult to interpret $(17,27)$. Second, there appears to be a region of TonB that cannot be analyzed by in vivo photocrosslinking. We previously used targeted amber mutations in $f e p A$ and in exbD to guide insertion of the photocrosslinkable amino acid $\mathrm{pBpa}$ and generate crosslinks at unknown sites in TonB in vivo. In those studies, both the fep $A$ and 
734 exbD amber mutations fully incorporate the $\mathrm{pBpa}$ and result in full-length proteins $735(40,65)$.

736

We were hopeful that a reciprocal approach using targeted amber mutations

737 in $\operatorname{ton} B$ at the carboxy terminal aromatic residues would be fruitful, however, we

738 were thwarted by failure to incorporate sufficient $\mathrm{pBpa}$, except small amounts and

739 only after highest overexpression, such that $\sim 85-100 \%$ of the TonB was present as

740 the truncated amber mutant form or its degradation product. Given the dominant

741 negative gene dosage effect of $\operatorname{ton} B$ overexpression, the high level of incomplete

742 TonB fragments would have obscured meaningful interpretations $(3,66)$. There

743 may be something unusual about this particular region of ton $B$ during translation

744 since we have been able to successfully incorporate $\mathrm{pBpa}$ into ton $B$ at engineered

745 amber sites in the transmembrane domain (Postle and Guzek, unpublished data).

$747 \quad$ Surveillance of FepA periplasmic $\beta$-strand turns

TonB binds to transporters whether or not it is "energized", although it is

750 still not clear what that term means mechanistically. For example, inactive TonB

751 H20A formaldehyde-crosslinks to FepA in vivo (18) and purified inactive carboxy

752 terminal domains of TonB bind with varying affinities to purified transporters in

753 vitro $(19,21,22)$. Here we identified the first in vivo interactions between the 
754 TonB amphipathic helix and the FepA $\beta$-strand turns, the majority of which

755 appeared to represent interactions with inactive TonB.

756 The periplasmic $\beta$-strand turns are candidates for one or more TonB-FepA

757 binding sites since they protrude more deeply into the periplasm than the face of

758 the cork does (Fig. 11). We chose Cys substitutions at Asp or Glu residues (and

759 Pro243) in the periplasmic $\beta$-strand turns as most likely to be required for FepA

760 activity and were surprised they were all functional. The fact that they had little to

761 no effect on activity, builds on and confirms a tolerance to mutation that generally

762 characterizes TBDTs $(36,40)$, where only certain structurally disruptive mutations

763 such as a Leu-to-Pro mutation in the TonB box, its complete deletion, or Arg-to-

764 Pro in the lock region residue 75 lead to TBDT inactivation $(4,15,55)$. it was impervious to the presence of the inactivating TonB H20A substitution. In contrast, TonB R212C formed complexes with Cys residues in several $\beta$-strand turns, the majority of which decreased if TonB was inactivated by the H20A mutation. 
774 this study by FepA D185C and P243C (56). We did not observe interaction of

775 TonB with D185, but we did observe R204C, N208C and R212C interacting with

776 P243C; interactions by N208C and R212C were both H20-dependent.

777 Considering all the results, this study demonstrated that the hydrophilic face

778 of the essential TonB amphipathic helix was used for contacts throughout FepA. It

779 also validated the idea that, in vivo, there are certain TonB-FepA contacts made by

780 active TonB, with a different set of contacts that do not lead to energy transduction

781 events. The contacts made by inactive "unenergized" TonB, some of which were

782 quite abundant, could be consistent with membrane surveillance and

783 conformational sampling $(27,46)$ where TonB discriminates between a TBDT and

784 a porin, or searches for a ligand-loaded TBDT (67). Because the ligand

785 enterochelin was present throughout the experiments here, it will be important to

786 determine which TonB-FepA interaction sites are ligand-dependent. If any in vivo

787 TonB-FepA interactions are H20-dependent as well as ligand-dependent, they

788 would constitute candidates important for energy transduction. 
791

792

793

794

795

796

797

798

799

800

801

802

803

804

805

806

807

808

809

810

\section{METHODS AND MATERIALS}

\section{Bacterial strains \& plasmids}

The strains and plasmids used in this study are listed in Table 2 . All bacteria are derivatives of Escherichia coli K-12 strain W3110. KP1491 was constructed by $\mathrm{P} 1_{\text {vir }}$ transduction of the $\Delta(\operatorname{ton} B, P 14):$ blaM cassette from KP1477 into KP1489 (W3110 $\Delta f e p A)$.

Plasmids pKP1858 and pKP1859 were created from pKP476 and pKP372 respectively, using polymerase chain reaction (PCR)-based site-directed mutagenesis as previously described (26) to create C18G substitutions in both plasmids. The majority of plasmids encoding ton $B$ mutants were derived from pKP1362 (tonB C18G), which was constructed by cloning tonB C18G from pKP568 into the Sph1 site of pPro33, allowing for expression from the propionate promoter (68). Plasmids encoding fepA mutants were derived from pKP515 where $f e p A$ is expressed from the arabinose promoter in pBAD24 (11). Mutations were engineered through PCR-based site-directed mutagenesis as previously described (26). The coding region of each engineered mutant gene was confirmed through Sanger sequencing at the Pennsylvania State University Nucleic Acid Facility.

\section{Growth media and culture conditions}


$81340 \mu \mathrm{g} \mathrm{ml}^{-1}$ of tryptophan, $4 \mu \mathrm{g} \mathrm{ml}^{-1}$ of thiamine, $1 \mathrm{mM} \mathrm{MgSO}$ and $0.5 \mathrm{mM} \mathrm{CaCl}_{2}$.

814 For disulfide-crosslinking, the M9 minimal salts medium was further

815 supplemented with $10 \mu \mathrm{M}$ Fe (as ferric chloride). For $\left[{ }^{55} \mathrm{Fe}\right]$-enterochelin transport

816 assays, the M9 minimal salts medium was supplemented with $1.85 \mu \mathrm{M} \mathrm{Fe}$ (as

817 ferric chloride) as well as $40 \mu \mathrm{g} \mathrm{ml}^{-1}$ of tyrosine and $40 \mu \mathrm{g} \mathrm{ml}^{-1}$ of phenylalanine to

818 facilitate growth of $\operatorname{aroB}$ strains on which they were performed (69).

819 Chloramphenicol at $34 \mu \mathrm{g} \mathrm{ml}^{-1}$ and ampicillin at $100 \mu \mathrm{g} \mathrm{ml}^{-1}$ were used to maintain

821 following concentrations with sodium propionate to achieve chromosomal levels;

822 TonB C18G (10 mM), TonB C18G M201C (10 mM), TonB C18G R204C (0.5

$823 \mathrm{mM})$, TonB C18G V206C (10 mM), TonB C18G N208C (10 mM), TonB C18G

824 A209C (10 mM), TonB C18G R212C (1 mM), TonB C18G R214C (10 mM), all

825 TonB C18G H20A cysteine substitutions (15 mM). FepA cysteine substitutions

826 were not induced as the base expression level approximated chromosomally

827 encoded FepA levels in cells grown with $1.85 \mu \mathrm{M}$ Fe. 
831 described previously (43) with some modifications. Strain KP1344 containing plasmids pKP1858 or pKP1859 was grown as described above, in the presence of $0.002 \%$ arabinose and $0.1 \%$ arabinose respectively, to mid exponential phase.

Cells were harvested and lysed by French pressure cell at $4^{\circ} \mathrm{C}$. The cell lysate supernatant was applied to the top of the sucrose gradient and centrifuged in a then immunoblotted with TonB 4F1 monoclonal antibodies (71).

\section{In vivo formaldehyde crosslinking}


850

851

852

853

854

855

856

857

858

859

860

861

862

863

864

865

866

867

868

869

usual concentration) and heated for 5 minutes at $60^{\circ} \mathrm{C}$. The samples were

electrophoresed on 11\% SDS-polyacrylamide gels and then immunoblotted with

TonB 4F1 monoclonal antibodies or anti-ExbD polyclonal antibodies $(32,71)$.

\section{In vivo disulfide crosslinking}

KP1491 harboring pairwise combinations of plasmid-encoded TonB and plasmid encoded FepA were subcultured 1:100 from saturated LB cultures into supplemented M9 minimal salts medium and grown with appropriate antibiotics to $\mathrm{A}_{550}=0.45 .0 .4 \mathrm{OD} \mathrm{ml}^{-1}$ of cells were harvested by centrifugation and precipitated with an equal volume of $4^{\circ} \mathrm{C} 20 \%$ TCA to stop the proteolysis of TonB that occurs in LSB at $95^{\circ} \mathrm{C}$ when TCA is not used (72). The TCA-precipitated pellets were boiled at $95^{\circ} \mathrm{C}$ for 10 minutes in $100 \mu 1$ of LSB with $50 \mathrm{mM}$ iodoacetamide to block any remaining free cysteines to prevent in vitro disulfide crosslinking. All samples were analyzed on 9\% SDS-PAGE gels and followed by immunoblot analysis with TonB 4F1 monoclonal antibody and FepA polyclonal antibodies (32).

To eliminate the possibility that disulfide crosslinks formed due to the presence of TCA during cell harvesting, the efficiency of crosslinking was compared with and without TCA precipitation. Upon harvesting cells were pelleted without TCA. The cell pellets were boiled at $95^{\circ} \mathrm{C}$ for 10 minutes in 100 
$870 \mu$ of LSB with $50 \mathrm{mM}$ iodoacetamide. TCA slightly enhanced the recovery of both

871 crosslinked complexes, which also still formed in the absence of TCA and the

872 monomer such that levels of crosslinking were proportional to the controls with

873 and without TCA (data not shown).

874

875

$\left[{ }^{55} \mathrm{Fe}\right]-$ enterochelin transport

876

TonB and FepA with individual Cys substitutions were assessed for their

877 initial rates of enterochelin (Sigma-Aldrich) transport as described previously (40).

878 FepA constructs were assayed in KP1490 (W3110 aroB $\Delta$ fepA) whereas TonB

879 constructs were assayed in strain KP1406 (W3110 aroB $\Delta($ tonB, P14)::blaM).

880 Enterochelin is the sole siderophore synthesized and secreted by E. coli K12. The

$881 a r o B$ mutation prevents enterochelin synthesis and the synthesis of any

882 intermediates that could interfere in the assay (69).

883

884

885

ACKNOWLEDGEMENTS

886

We thank Ryan Guzek for analysis of the TonB pBpa substitutions and

888 construction of pKP1836 and pKP1837; Bradley Spicer for construction of

889 pKP1382, pKP1400, pKP1403, pKP1427, FepA Cys substitutions in the TonB 
890 box, and analyzing effects of a $d s b A$ strain on TonB triplet homodimer formation;

891 Suzanne Wardell for construction of pKP372; Shaima El Mowafi for construction 892 of pKP1369 and pKP1370; Siti Kamarudin for construction of pKP1858, 893 pKP1859, and pKP1861; Surendran Devanathan for construction of KP1491;

894 Cheryl Swayne for construction of pKP1362; Raka Ghosh for construction of 895 pKP1581; Yu-An Liu for construction of pKP1361; Larissa Goldman for 896 construction of pKP1506; Gui Teng Chua for construction of pKP1841, pKP1850, 897 and pKP1854; Elizabeth McFadden for the formaldehyde crosslinking experiment 898 on the TonB double Ala variants, and Glenn Hwang for construction of pKP2299, 899 pKP2303, pKP2304, the analysis of amphipathic helix Cys substitutions 900 interactions with the FepA TonB box and excellent technical assistance. We thank 901 the E. coli Genetic Stock Center and the Jonathan Beckwith lab for their $d s b A:$ kan 902 strain, RI90. Support from NIGMS grant GM112710 is gratefully acknowledged. 
904

905

906

907

908

909

910

911

912

913

914

915

916 917 5. Davies JK, Reeves P. 1975. Genetics of resistance to colicins in

918

919

920

921

922

\section{REFERENCES}

1. Nikaido H. 2003. Molecular basis of bacterial outer membrane permeability revisited. Microbiol Mol Biol Rev 67:593-656.

2. Noinaj N, Guillier M, Barnard TJ, Buchanan SK. 2010. TonB-dependent transporters: regulation, structure, and function. Annu Rev Microbiol 64:43-60.

3. Fischer E, Günter K, Braun V. 1989. Involvement of ExbB and TonB in transport across the outer membrane of Escherichia coli: phenotypic complementation of exb mutants by overexpressed ton $B$ and physical stabilization of TonB by ExbB. J. Bacteriol. 171:5127-5134.

4. Cadieux N, Kadner RJ. 1999. Site-directed disulfide bonding reveals an interaction site between energy-coupling protein TonB and BtuB, the outer membrane cobalamin transporter. Proc. Natl. Acad. Sci. USA 96:10673-10678. Escherichia coli K-12: cross-resistance among colicins of group B. J. Bacteriol. 123:96-101.

6. Pugsley AP, Reeves P. 1976. Increased production of the outer membrane receptors for colicins B, D and M by Escherichia coli under iron starvation.

Biochem. Biophys. Res. Commun. 70:846-853. 
9237 Frost GE, Rosenberg H. 1975. Relationship between the tonB locus and 924 iron transport in Escherichia coli. J. Bacteriol 124:704-712.

925 8. Gresock MG, Savenkova MI, Larsen RA, Ollis AA, Postle K. 2011.

926 Death of the TonB shuttle hypothesis. Front. Microbiol. 2:206.

927 9. Ma L, Kaserer W, Annamalai R, Scott DC, Jin B, Jiang X, Xiao Q,

928 Maymani H, Massis LM, Ferreira LC, Newton SM, Klebba PE. 2007.

929 Evidence of ball-and-chain transport of ferric enterobactin through FepA. J. Biol.

930 Chem. 282:397-406.

931 10. Klebba PE, Newton SMC, Six DA, Kumar A, Yang T, Nairn BL,

932 Munger C, Chakravorty S. 2021. Iron acquisition systems of Gram-negative

933 bacterial pathogens define TonB-dependent pathways to novel antibiotics. Chem.

934 Rev. 121:5193-5239.

935 11. Vakharia HL, Postle K. 2002. FepA with globular domain deletions lacks

936 activity. J. Bacteriol. 184:5508-5512.

937 12. Devanathan S, Postle K. 2007. Studies on colicin B translocation: FepA is 938 gated by TonB. Mol. Microbiol. 65:441-453.

939 13. Udho E, Jakes KS, Finkelstein A. 2012. TonB-dependent transporter FhuA 940 in planar lipid bilayers: partial exit of its plug from the barrel. Biochemistry $941 \quad 51: 6753-6759$. 
942 14. Hickman SJ, Cooper REM, Bellucci L, Paci E, Brockwell DJ. 2017.

943 Gating of TonB-dependent transporters by substrate-specific forced remodelling.

$944 \quad$ Nat. Commun. 8:14804.

945 15. Gudmundsdottir A, Bell PE, Lundrigan MD, Bradbeer C, Kadner RJ.

946 1989. Point mutations in a conserved region (TonB Box) of the Escherichia coli

947 outer membrane BtuB protein affect vitamin B12 transport. J. Bacteriol. 171:6526-

$948 \quad 6533$.

949 16. Ogierman M, Braun V. 2003. Interactions between the outer membrane

950 ferric citrate transporter FecA and TonB: studies of the FecA TonB box. J.

951 Bacteriol. 185:1870-1885.

952 17. Ghosh J, Postle K. 2005. Disulphide trapping of an in vivo energy-

953 dependent conformation of Escherichia coli TonB protein. Mol. Microbiol.

$954 \quad 55: 276-288$.

955 18. Ollis AA, Manning M, Held KG, Postle K. 2009. Cytoplasmic membrane

956 protonmotive force energizes periplasmic interactions between ExbD and TonB.

957 Mol. Microbiol. 73:466-481.

958 19. Koedding J, Howard SP, Kaufman L, Polzer P, Lustig A, Welte W.

959 2004. Dimerization of TonB is not essential for its binding to the outer membrane

960 siderophore receptor FhuA of E. coli. J. Biol. Chem. 279:9978-9986. 
961 20. Pawelek PD, Croteau N, Ng-Thow-Hing C, Khursigara CM, Moiseeva

962 N, Allaire M, Coulton JW. 2006. Structure of TonB in complex with FhuA, $E$.

963 coli outer membrane receptor. Science 312:1399-1402.

964 21. Khursigara CM, De Crescenzo G, Pawelek PD, Coulton JW. 2004.

965 Enhanced binding of TonB to a ligand-loaded outer membrane receptor. Role of

966 the oligomeric state of TonB in formation of a functional FhuA-TonB complex. J.

967 Biol. Chem. 279:7405-7412.

968 22. Freed DM, Lukasik SM, Sikora A, Mokdad A, Cafiso DS. 2013.

969 Monomeric TonB and the Ton Box are required for the formation of a high-affinity

970 transporter-TonB complex. Biochemistry 52:2638-2648.

971 23. Postle K, Skare JT. 1988. Escherichia coli TonB protein is exported from

972 the cytoplasm without proteolytic cleavage of its amino terminus. J. Biol. Chem.

973 263:11000-11007.

974 24. Larsen RA, Wood GE, Postle K. 1993. The conserved proline-rich motif is

975 not essential for energy transduction by Escherichia coli TonB protein. Mol

976 Microbiol 10:943-953.

977 25. Skare JT, Roof SK, Postle K. 1989. A mutation in the amino terminus of a

978 hybrid TrpC-TonB protein relieves overproduction lethality and results in

979 cytoplasmic accumulation. J. Bacteriol. 171:4442-4447. 
26. Vakharia-Rao H, Kastead KA, Savenkova MI, Bulathsinghala CM,

Postle K. 2007. Deletion and substitution analysis of the Escherichia coli TonB

982

Q160 region. J. Bacteriol. 189:4662-4670.

27. Postle K, Kastead KA, Gresock MG, Ghosh J, Swayne CD. 2010. The

TonB dimeric crystal structures do not exist in vivo. mBio 1:e00307-00310.

985

28. Swayne C, Postle K. 2011. Taking the Escherichia coli TonB

986

transmembrane domain "Offline"? Non-protonatable Asn substitutes fully for

TonB His20. J. Bacteriol. 193:3693-3701.

988

29. Ghosh J, Postle K. 2004. Evidence for dynamic clustering of carboxy-

terminal aromatic amino acids in TonB-dependent energy transduction. Mol.

990

Microbiol. 51:203-213.

991

30. Cascales E, Buchanan SK, Duché D, Kleanthous C, Lloubes R, Postle K,

992

Riley M, Slatin S, Cavard D. 2007. Colicin Biology. Micro. Mol. Biol. Rev.

71:158-229.

994 31. Gresock MG, Kastead KA, Postle K. 2015. From homodimer to

995 heterodimer and back: Elucidating the TonB energy transduction cycle. J.

996 Bacteriol. 197:3433-3445.

997 32. Higgs PI, Larsen RA, Postle K. 2002. Quantitation of known components

998 of the Escherichia coli TonB-dependent energy transduction system: TonB, ExbB,

999 ExbD, and FepA. Mol. Microbiol. 44:271-281. 
$100033 . \quad$ Kadner RJ, Heller KJ. 1995. Mutual inhibition of cobalamin and 1001 siderophore uptake systems suggests their competition for TonB function. J.

1002 Bacteriol. 177:4829-4835.

1003 34. Ollis AA, Postle K. 2011. The same periplasmic ExbD residues mediate in 1004 vivo interactions between ExbD homodimers and ExbD-TonB heterodimers. J.

1005 Bacteriol. 193:6852-6863.

1006 35. Ollis AA, Postle K. 2012. Identification of functionally important TonB1007 ExbD periplasmic domain interactions in vivo. J. Bacteriol. 194:3078-3087.

1008 36. Endriss F, Braun M, Killmann H, Braun V. 2003. Mutant analysis of the 1009 Escherichia coli FhuA protein reveals sites of FhuA activity. J. Bacteriol.

1010 185:4683-4692.

1011 37. Larsen RA, FosterHartnett D, McIntosh MA, Postle K. 1997. Regions of 1012 Escherichia coli TonB and FepA proteins essential for in vivo physical 1013 interactions. J. Bacteriol. 179:3213-3221.

1014 38. Evans JS, Levine BA, Trayer IP, Dorman CJ, Higgins CF. 1986.

1015 Sequence-imposed structural constraints in the TonB protein of E. coli. FEBS Lett. $1016 \quad$ 208:211-216.

1017 39. Seliger SS, Mey AR, Valle AM, Payne SM. 2001. The two TonB systems 1018 of Vibrio cholerae: redundant and specific functions. Mol. Microbiol. 39:801-812. 
1019 40. Gresock MG, Postle K. 2017. Going outside the TonB Box: Identification 1020 of novel FepA-TonB interactions in vivo. J. Bacteriol. 199:e00649-00616.

1021 41. Postle K, Good RF. 1983. DNA sequence of the Escherichia coli tonB 1022 gene. Proc. Natl. Acad. Sci. USA 80:5235-5239.

1023 42. Larsen RA, Chen GJ, Postle K. 2003. Performance of standard phenotypic 1024 assays for TonB activity, as evaluated by varying the level of functional, wild-type 1025 TonB. J. Bacteriol. 185:4699-4706.

1026 43. Letain TE, Postle K. 1997. TonB protein appears to transduce energy by 1027 shuttling between the cytoplasmic membrane and the outer membrane in Gram1028 negative bacteria. Mol. Microbiol. 24:271-283.

1029 44. Larsen RA, Deckert GE, Kastead KA, Devanathan S, Keller KL, Postle 1030 K. 2007 . His20 provides the sole functionally significant side chain in the essential 1031 TonB transmembrane domain. J. Bacteriol. 189:2825-2833.

1032 45. Shultis DD, Purdy MD, Banchs CN, Wiener MC. 2006. Outer membrane 1033 active transport: structure of the BtuB:TonB complex. Science 312:1396-1399.

1034 46. Kaserer WA, Jiang X, Xiao Q, Scott DC, Bauler M, Copeland D, 1035 Newton SM, Klebba PE. 2008. Insight from TonB hybrid proteins into the 1036 mechanism of iron transport through the outer membrane. J. Bacteriol. 190:400110374016. 
1038 47. Higgs PI, Letain TE, Merriam KK, Burke NS, Park H, Kang C, Postle

K. 2002. TonB interacts with nonreceptor proteins in the outer membrane of

1041 48. Heller KJ, Kadner RJ, Günter K. 1988. Suppression of the btuB451

1042 mutation by mutations in the $\operatorname{ton} B$ gene suggests a direct interaction between TonB 1043 and TonB-dependent receptor proteins in the outer membrane of Escherichia coli.

1044 Gene 64: 147-153.

1045 49. Bell PE, Nau CD, Brown JT, Konisky J, Kadner RJ. 1990. Genetic 1046 suppression demonstrates direct interaction of TonB protein with outer membrane 1047 transport proteins in Escherichia coli. J. Bacteriol. 172:3826-3829.

1048 50. Cadieux N, Bradbeer C, Kadner RJ. 2000. Sequence changes in the ton 1049 box region of BtuB affect its transport activities and interaction with TonB protein.

1050

1051

1052

1053

1054

1055

1056

J. Bacteriol. 182:5954-5961.

51. Mende J, Braun V. 1990. Import-defective colicin B derivatives mutated in the TonB box. Mol. Microbiol. 4:1523-1533.

52. Majumdar A, Trinh V, Moore KJ, Smallwood CR, Kumar A, Yang T, Scott DC, Long NJ, Newton SM, Klebba PE. 2020. Conformational rearrangements in the N-domain of Escherichia coli FepA during ferric enterobactin transport. J. Biol. Chem. 295:4974-4984. 
1057 53. Chimento DP, Kadner RJ, Wiener MC. 2005. Comparative structural 1058 analysis of TonB-dependent outer membrane transporters: implications for the 1059 transport cycle. Proteins 59:240-251.

1060 54. Chakraborty R, Storey E, van der Helm D. 2006. Molecular mechanism 1061 of ferricsiderophore passage through the outer membrane receptor proteins of 1062 Escherichia coli. Biometals 20:263-274.

1063 55. Barnard TJ, Watson ME, Jr., McIntosh MA. 2001. Mutations in the 1064 Escherichia coli receptor FepA reveal residues involved in ligand binding and 1065 transport. Mol. Microbiol. 41:527-536.

1066 56. Carter DM, Gagnon JN, Damlaj M, Mandava S, Makowski L, Rodi DJ, 1067 Pawelek PD, Coulton JW. 2006. Phage display reveals multiple contact sites 1068 between FhuA, an outer membrane receptor of Escherichia coli, and TonB. J. Mol. 1069 Biol. 357:236-251.

1070 57. Nilaweera TD, Nyenhuis DA, Cafiso DS. 2021. Structural intermediates 1071 observed only in intact Escherichia coli indicate a mechanism for TonB-dependent 1072 transport. Elife 10:e68548.

1073 58. Larsen RA, Letain TE, Postle K. 2003. In vivo evidence of TonB shuttling 1074 between the cytoplasmic and outer membrane in Escherichia coli. Mol. Microbiol. $1075 \quad 49: 211-218$. 
1076 59. Bitter W, Tommassen J, Weisbeek PJ. 1993. Identification and

1077 characterization of the exbB, exbD and tonB Genes of Pseudomonas putida

1078 WCS358 - Their involvement in ferric pseudobactin transport. Mol. Microbiol.

$1079 \quad 7: 117-130$.

1080 60. Buchanan SK, Smith BS, Venkatramani L, Xia D, Esser L, Palnitkar M,

1081 Chakraborty R, van der Helm D, Deisenhofer J. 1999. Crystal structure of the

1082 outer membrane active transporter FepA from Escherichia coli. Nat. Struct. Biol.

1083 6:56-63.

1084 61. Hilsenbeck JL, Park H, Chen G, Youn B, Postle K, Kang C. 2004.

1085 Crystal structure of the cytotoxic bacterial protein colicin B at 2.5 A resolution.

1086 Mol. Microbiol. 51:711-720.

1087 62. White P, Joshi A, Rassam P, Housden NG, Kaminska R, Goult JD,

1088 Redfield C, McCaughey LC, Walker D, Mohammed S, Kleanthous C. 2017.

1089 Exploitation of an iron transporter for bacterial protein antibiotic import. Proc.

1090 Natl. Acad. Sci. USA 114:12051-12056.

1091 63. Hullmann J, Patzer SI, Romer C, Hantke K, Braun V. 2008. Periplasmic

1092 chaperone FkpA is essential for imported colicin M toxicity. Mol. Microbiol.

1093 69:926-937.

1094 64. Landeta C, Boyd D, Beckwith J. 2018. Disulfide bond formation in

1095 prokaryotes. Nat. Microbiol. 3:270-280. 
1096 65. Kopp DR, Postle K. 2020. The intrinsically disordered region of ExbD is

1097 required for signal transduction. J. Bacteriol. 202:e00687-00619.

1098 66. Mann BJ, Holroyd CD, Bradbeer C, Kadner RJ. 1986. Reduced activity

1099 of TonB-dependent functions in strains of Escherichia coli. FEMS Lett. 33:255-

1100260.

1101 67. Larsen RA, Thomas MG, Postle K. 1999. Protonmotive force, ExbB and

1102 ligand-bound FepA drive conformational changes in TonB. Mol. Microbiol.

1103 31:1809-1824.

1104 68. Lee SK, Keasling JD. 2005. A propionate-inducible expression system for 1105 enteric bacteria. Appl. Environ. Microbiol. 71:6856-6862.

1106 69. Postle K. 2007. TonB system, in vivo assays and characterization. Methods 1107 Enzymol. 422:245-269.

1108 70. Laemmli UK. 1970. Cleavage of structural proteins during the assembly of

1109 the head of bacteriophage T4. Nature 227:680-685.

1110 71. Larsen RA, Myers PS, Skare JT, Seachord CL, Darveau RP, Postle K.

1111 1996. Identification of TonB homologs in the family Enterobacteriaceae and

1112 evidence for conservation of TonB-dependent energy transduction complexes. J.

1113 Bacteriol. 178:1363-1373.

1114 72. Skare JT, Postle K. 1991. Evidence for a TonB-dependent energy

1115 transduction complex in Escherichia coli. Mol. Microbiol. 5:2883-2890. 
1116 73. Hill CW, Harnish BW. 1981. Inversions between ribosomal RNA genes of

1117 Escherichia coli. Proc. Natl. Acad. Sci. USA 78:7069-7072.

1118 74. Baker KR, Postle K. 2013. Mutations in Escherichia coli ExbB

1119 transmembrane domains identify scaffolding and signal transduction functions and

1120 exclude participation in a proton pathway. J. Bacteriol. 195:2898-2911.

1121 75. Chang C, Mooser A, Pluckthun A, Wlodawer A. 2001. Crystal structure

1122 of the dimeric C-terminal domain of TonB reveals a novel fold. J. Biol. Chem.

$1123 \quad$ 276:27535-27540.

1124 76. Peacock SR, Weljie AM, Peter Howard S, Price FD, Vogel HJ. 2005.

1125 The solution structure of the C-terminal domain of TonB and interaction studies

1126 with TonB box peptides. J. Mol. Biol. 345:1185-1197.

1127 77. Symmons MF, Bokma E, Koronakis E, Hughes C, Koronakis V. 2009.

1128 The assembled structure of a complete tripartite bacterial multidrug efflux pump.

1129 Proc. Natl. Acad. Sci. USA 106:7173-7178.

$113078 . \quad$ Drozdetskiy A, Cole C, Procter J, Barton GJ. 2015. JPred4: a protein

1131 secondary structure prediction server. Nucl. Acids Res. 43:W389-394. 


\section{TABLES}

1134 Table 1: [ $\left.{ }^{55} \mathrm{Fe}\right]-e n t e r o c h e l i n$ transport activities of TonB and FepA Cys substitutions

\begin{tabular}{cc}
\hline Mutant & $\begin{array}{c}\text { Percent activity (\%) relative } \\
\text { to "wild-type" }\end{array}$ \\
\hline TonB C18G & 100 \\
TonB C18G Q160C & 113 \\
TonB C18G M201C & 95 \\
TonB C18G R204C & 107 \\
TonB C18G V206C & 71 \\
TonB C18G N208C & 76 \\
TonB C18G A209C & 65 \\
TonB C18G R212C & 96 \\
TonB C18G R214C & 75 \\
TonB C18G H20A Q160C & 5 \\
TonB C18G H20A M201C & 3 \\
TonB C18G H20A R204C & 3 \\
TonB C18G H20A N208C & 3 \\
\hline FepA wild-type & \\
\hline & \\
\hline & \\
\hline & \\
\hline
\end{tabular}




\begin{tabular}{|c|c|}
\hline FepA V16C & 91 \\
\hline FepA L23C & 79 \\
\hline FepA S29C & 98 \\
\hline FepA T32C & 92 \\
\hline FepA A33C & 89 \\
\hline FepA D34C & 115 \\
\hline FepA E35C & 86 \\
\hline FepA A42C & 77 \\
\hline FepA S46C & 76 \\
\hline FepA T51C & 85 \\
\hline FepA G54C & 86 \\
\hline FepA R75C & 40 \\
\hline FepA L85C & 107 \\
\hline FepA V91C & 84 \\
\hline FepA S92C & 101 \\
\hline FepA S112C & 105 \\
\hline FepA E120C & 83 \\
\hline FepA V124C & 102 \\
\hline FepA R126C & 46 \\
\hline FepA A131C & 72 \\
\hline
\end{tabular}




\begin{tabular}{|c|c|}
\hline FepA V142C & 78 \\
\hline FepA I145C & 84 \\
\hline FepA E152C & 62 \\
\hline FepA D185C & 118 \\
\hline FepA P243C & 106 \\
\hline FepA D298C & 126 \\
\hline FepA D356C & 115 \\
\hline FepA D422C & 109 \\
\hline FepA D455C & 121 \\
\hline FepA E511C & 92 \\
\hline FepA D519C & 136 \\
\hline FepA E567C & 65 \\
\hline FepA E576C & 134 \\
\hline FepA D618C & 104 \\
\hline FepA D664C & 105 \\
\hline FepA T722C & 60 \\
\hline
\end{tabular}

1135 
1138 Table 2: Strains and plasmids

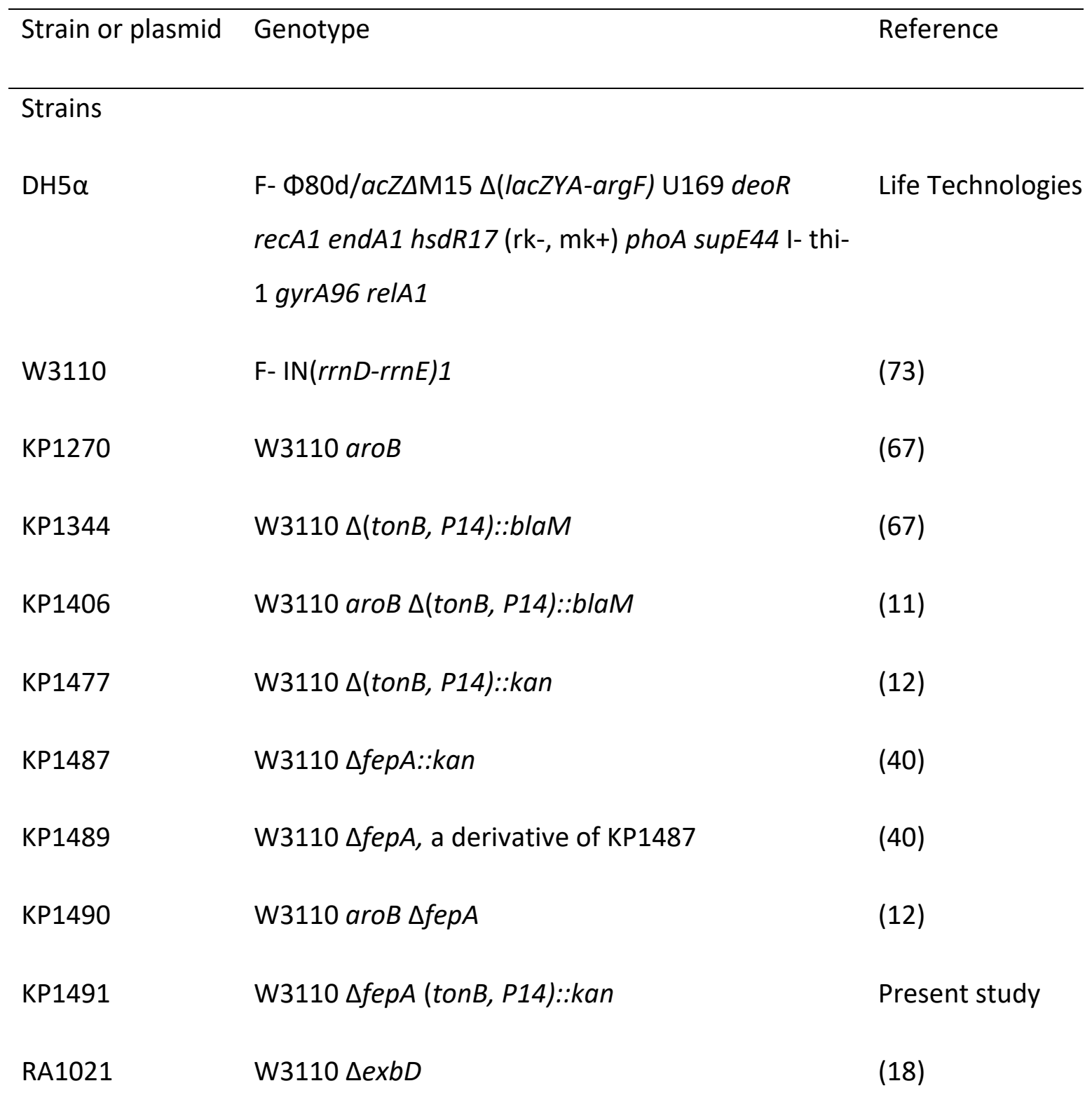

TonB Plasmids

pKP325 Wild-type TonB in pACYC184

pKP372 TonB amphipathic helix frameshift in pKP325 Present study

pKP1859 pKP372, C18G Present study 


\begin{tabular}{|c|c|c|}
\hline pKP442 & Wild-type TonB in pKP325, silent Xhol site & (29) \\
\hline pKP568 & pKP442 with TonB C18G & (29) \\
\hline pKP531 & pKP442 with TonB F202A, W213A & (29) \\
\hline pKP532 & pKP442 with TonB F202A, Y215A & (29) \\
\hline pKP476 & pKP442 with TonB $\Delta 199-216$ & Present study \\
\hline pKP1858 & pKP476, C18G & Present study \\
\hline pKP1362 & TonB C18G in pPro33 & Present study \\
\hline pKP1427 & TonB C18G Q160C, a derivative of pKP1362 & Present study \\
\hline pKP2303 & TonB C18G Q160C F202A, a derivative of pKP1427 & Present study \\
\hline pKP2304 & $\begin{array}{l}\text { TonB C18G Q160C F202A W213A, a derivative of } \\
\text { pKP2303 }\end{array}$ & Present study \\
\hline pKP1676 & TonB C18G M201C, a derivative of pKP1362 & Present study \\
\hline pKP1867 & TonB C18G R204C, a derivative of pKP1362 & Present study \\
\hline pKP1683 & TonB C18G V206C, a derivative of pKP1362 & Present study \\
\hline pKP1646 & TonB C18G N208C, a derivative of pKP1362 & Present study \\
\hline pKP1647 & TonB C18G A209C, a derivative of pKP1362 & Present study \\
\hline pKP1624 & TonB C18G R212C, a derivative of pKP1362 & Present study \\
\hline pKP1684 & TonB C18G R214C, a derivative of pKP1362 & Present study \\
\hline pKP1708 & TonB C18G H20A Q160C, a derivative of pKP1427 & Present study \\
\hline pKP1720 & TonB C18G H20A M201C, a derivative of pKP1676 & Present study \\
\hline
\end{tabular}




$\begin{array}{lll}\text { pKP1868 } & \text { TonB C18G H20A R204C, a derivative of pKP1645 } & \text { Present study } \\ \text { pKP1722 } & \text { TonB C18G H20A V206C, a derivative of pKP1683 } & \text { Present study } \\ \text { pKP1692 } & \text { TonB C18G H20A N208C, a derivative of pKP1646 } & \text { Present study } \\ \text { pKP2299 } & \text { TonB C18G H20A V209C, a derivative of pKP1647 } & \text { Present study } \\ \text { pKP1861 } & \text { TonB C18G H20A R212C, a derivative of pKP1624 } & \text { Present study } \\ \text { pKP1723 } & \text { TonB C18G H20A R214C, a derivative of pKP1684 } & \text { Present study }\end{array}$

FepA plasmids

pKP515 WT FepA in pBAD24

\begin{tabular}{|c|c|c|}
\hline pKP1410 & FepA D12C, a pKP515 derivative & Present study \\
\hline pKP718 & FepA T13C, a pKP515 derivative & $(12)$ \\
\hline pKP1411 & FepA I14C, a pKP515 derivative & Present study \\
\hline pKP1383 & FepA V15C, a pKP515 derivative & Present study \\
\hline pKP1384 & FepA V16C, a pKP515 derivative & Present study \\
\hline pKP1416 & FepA T17C, a pKP515 derivative & Present study \\
\hline pKP1626 & $\begin{array}{l}\text { FepA D34C, } \triangle \text { TonB box (residues 12-16), } \\
\text { derivative of pKP1583 }\end{array}$ & Present study \\
\hline pKP1627 & $\begin{array}{l}\text { FepA G54C, } \triangle \text { TonB box (residues 12-16), } \\
\text { derivative of pKP1382 }\end{array}$ & Present study \\
\hline pKP1582 & FepA L23C, a pKP515 derivative & Present study \\
\hline
\end{tabular}




\begin{tabular}{|c|c|c|}
\hline pKP719 & FepA S29C, a pKP515 derivative & $(12)$ \\
\hline pKP1577 & FepA T32C, a pKP515 derivative & Present study \\
\hline pKP728 & FepA A33C, a pKP515 derivative & $(12)$ \\
\hline pKP1583 & FepA D34C, a pKP515 derivative & Present study \\
\hline pKP1578 & FepA E35C, a pKP515 derivative & Present study \\
\hline pKP720 & FepA A42C, a pKP515 derivative & $(12)$ \\
\hline pKP729 & FepA S46C, a pKP515 derivative & $(12)$ \\
\hline pKP730 & FepA T51C, a pKP515 derivative & $(12)$ \\
\hline pKP1382 & FepA G54C, a pKP515 derivative & Present study \\
\hline pKP1400 & FepA R75C, a pKP515 derivative & Present study \\
\hline pKP1581 & FepA L85C, a pKP515 derivative & Present study \\
\hline pKP731 & FepA V91C, a pKP515 derivative & $(12)$ \\
\hline pKP721 & FepA S92C, a pKP515 derivative & $(12)$ \\
\hline pKP732 & FepA S112C, a pKP515 derivative & $(12)$ \\
\hline pKP1836 & FepA E120C, a pKP515 derivative & Present study \\
\hline pKP733 & FepA V124C, a pKP515 derivative & $(12)$ \\
\hline pKP1506 & FepA R126C, a pKP515 derivative & Present study \\
\hline pKP1854 & FepA A131C, a pKP515 derivative & Present study \\
\hline pKP1841 & FepA V142C, a pKP515 derivative & Present study \\
\hline pKP1837 & FepA I145C, a pKP515 derivative & Present study \\
\hline
\end{tabular}




\begin{tabular}{|c|c|c|}
\hline pKP1857 & FepA E152C, a pKP515 derivative & Present study \\
\hline pKP1369 & FepA D185C, a pKP515 derivative & Present study \\
\hline pKP1864 & FepA P243C, a pKP515 derivative & Present study \\
\hline pKP1361 & FepA D298C, a pKP515 derivative & Present study \\
\hline pKP1685 & FepA D356C, a pKP515 derivative & Present study \\
\hline pKP1726 & FepA D422C, a pKP515 derivative & Present study \\
\hline pKP1656 & FepA D455C, a pKP515 derivative & Present study \\
\hline pKP1609 & FepA E511C, a pKP515 derivative & Present study \\
\hline pKP1410 & FepA D519C, a pKP515 derivative & Present study \\
\hline pKP1610 & FepA E567C, a pKP515 derivative & Present study \\
\hline pKP1793 & FepA E576C, a pKP515 derivative & Present study \\
\hline pKP1370 & FepA D618C, a pKP515 derivative & Present study \\
\hline pKP1727 & FepA D664C, a pKP515 derivative & Present study \\
\hline pKP1850 & FepA T722C, a pKP515 derivative & Present study \\
\hline
\end{tabular}



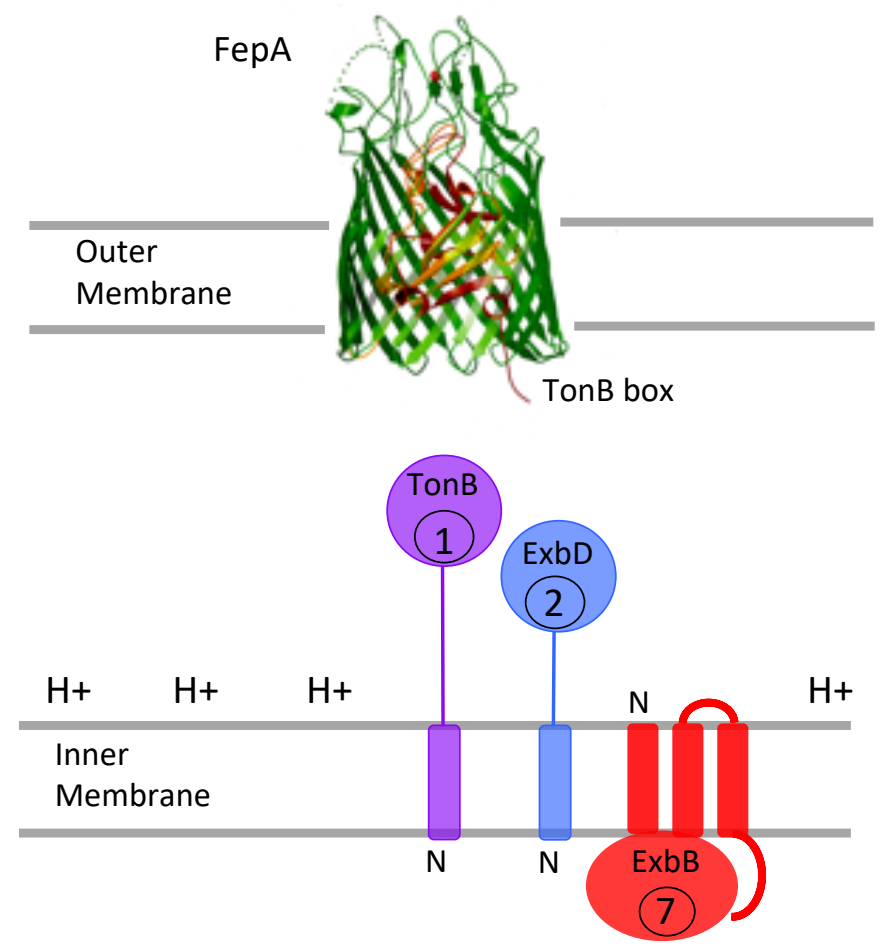

1143 Figure 1. The TonB system of Escherichia coli K12. The TonB-dependent

1144 transporter, FepA, is shown in the outer membrane. At its extreme amino terminus,

1145 the TonB box, the only known site of in vivo interaction with TonB, is shown

1146 protruding into the periplasm. The topologies and cellular ratios of the cytoplasmic

1147 membrane proteins TonB, ExbB and ExbD are shown in the cytoplasmic

1148 membrane. The protonmotive force gradient of the cytoplasmic membrane $(\mathrm{H}+)$ is

1149 shown. The crystal structure of FepA was solved by Buchanan et al. (60). 


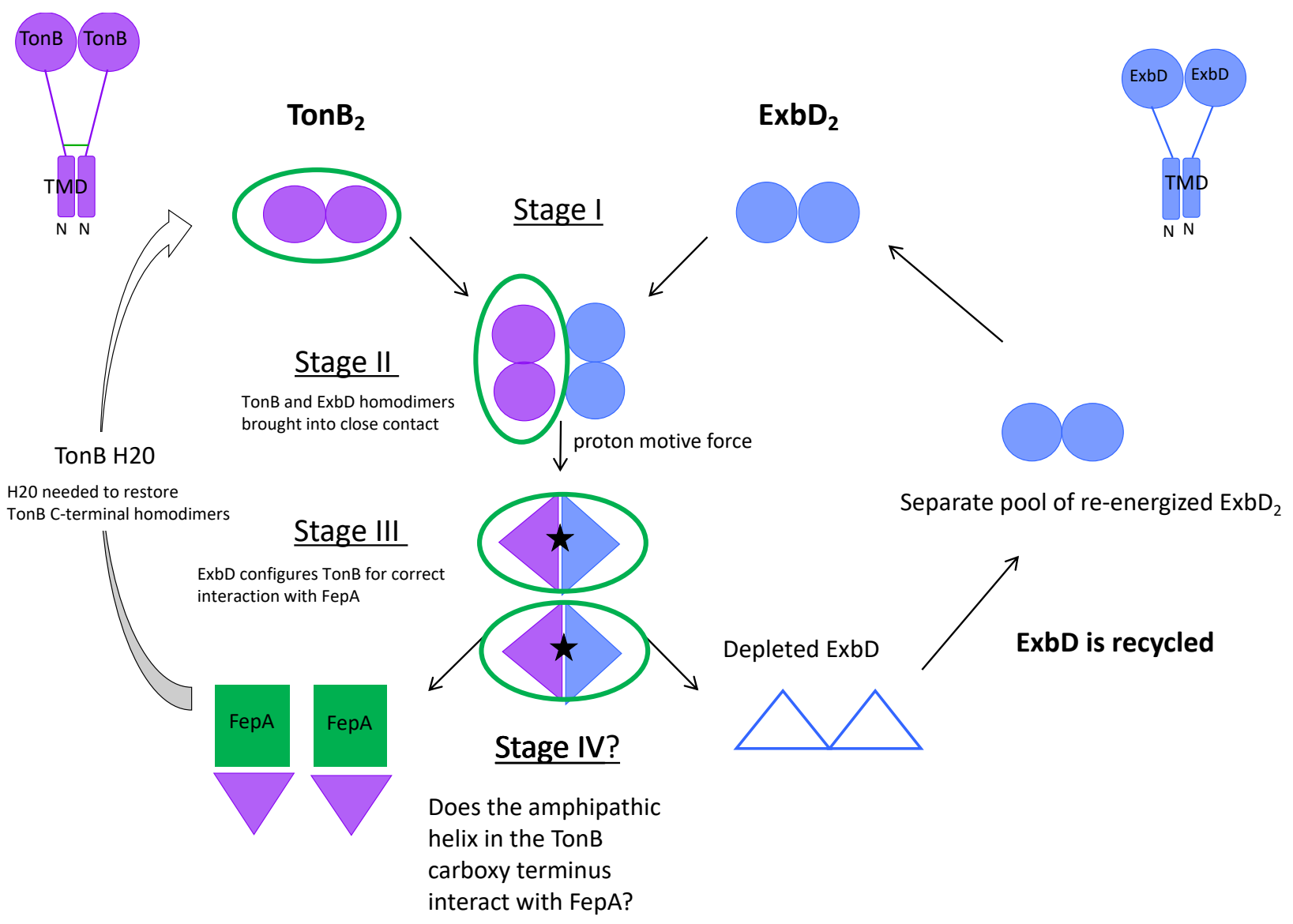

Figure 2. Key in vivo interactions of the TonB carboxy terminus during the

1158 in the cytoplasmic membrane with the bulk of the residues occupying the

1159 periplasmic space. Filled purple circles/triangles are TonB carboxy termini. Filled 
1162 terminus undergoing sequential protein-protein interactions (31). Interactions of

1163 the periplasmic carboxy-terminal domains of both TonB 2 and $\mathrm{ExbD}_{2} \mathrm{homodimers}$

1164 are shown. In Stage I, H20 in the TonB transmembrane domain is required for

1165 TonB carboxy termini to form obligatory homodimers through residues in and near

1166 the carboxy terminal amphipathic helix (residues 199-216), (17, 31). ExbB

1167 tetramers $\left(\mathrm{ExbB}_{4}\right)$ independently stabilize both TonB 2 and $\mathrm{ExbD}_{2}$, homodimerized

1168 through their carboxy termini, and are proposed to be the scaffolds upon which

$1169 \mathrm{~T}_{2} \mathrm{~B}_{2}$ and $\mathrm{ExbD}_{2}$ are independently assembled (ExbB 4 is not shown). In Stage II,

$1170 \mathrm{~T}_{2} \mathrm{~B}_{2}$ and $\mathrm{ExbD}_{2}$ homodimers are brought into close contact by ExbB tetramers

1171 but have not yet formed the heterodimers of the subsequent Stage. In Stage III, in

1172 the presence of the cytoplasmic membrane protonmotive force (PMF), the TonB 2

1173 and $\mathrm{ExbD}_{2}$ carboxy termini reassort to form two TonB-ExbD heterodimers. ExbD,

1174 which contains the sole potentially PMF-responsive residue (Asp25) among five

transmembrane domains that make up the TonB system, configures TonB correctly

1176 for a productive interaction with FepA [(28, 74); Jana and Postle, unpublished].

1177 This is necessary because inactive TonB also binds to TBDTs but without energy

1178 transduction, meaning that the correct configuration must be based on prior

1179 interaction with ExbD. Stage IV is binding of a monomeric carboxy terminus of

1180 TonB to FepA, such that active transport of the siderophore enterochelin across the

1181 outer membrane occurs. Notably, the TonB amphipathic helix makes important 
1182

1183

1184

1185

1186

1187

1188

1189

1190

1191

1192

1193

1194

1195

1196

1197

1198

1199

1200

1201

contacts with another TonB or ExbD in Stages I-III (green circles), however it has

never been tested for important contacts with FepA (green square). After a

transport event, H20 is required for re-formation of TonB dimers in Stage I.

$\mathrm{ExbD}_{2}$ is de-energized after this event (empty blue triangles) and needs to be

recycled. We speculate that $\mathrm{ExbD}_{2}$ moves in and out of the complex escorted by

ExbB tetramers. A separate pool of recycled $\mathrm{ExbB}_{4}-\mathrm{ExbD}_{2}$ is hypothesized to

exist to replenish Stage I ExbB4-ExbD homodimers. See (31) for a full explanation of the experimental basis for the model.

.




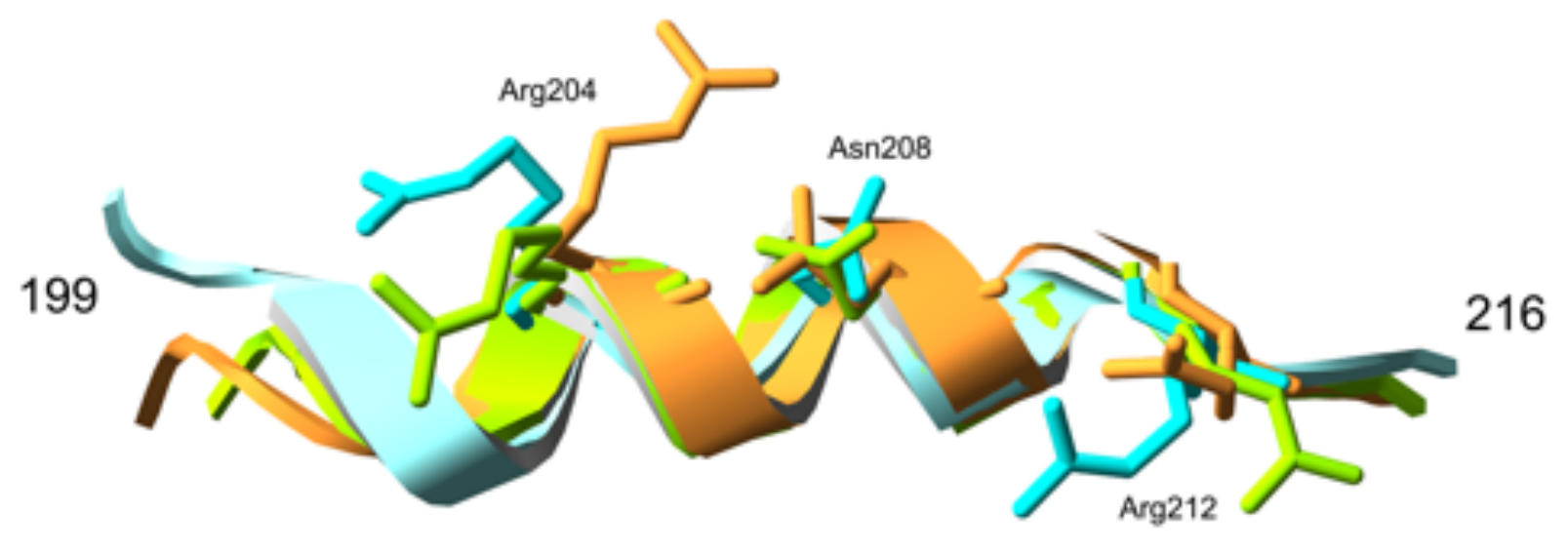

1209 is from Shultis et al. (45). The hydrophilic residues that, as Cys substitutions,

1210 interact with the FepA TonB box Cys substitutions in Fig. 7 are shown. 


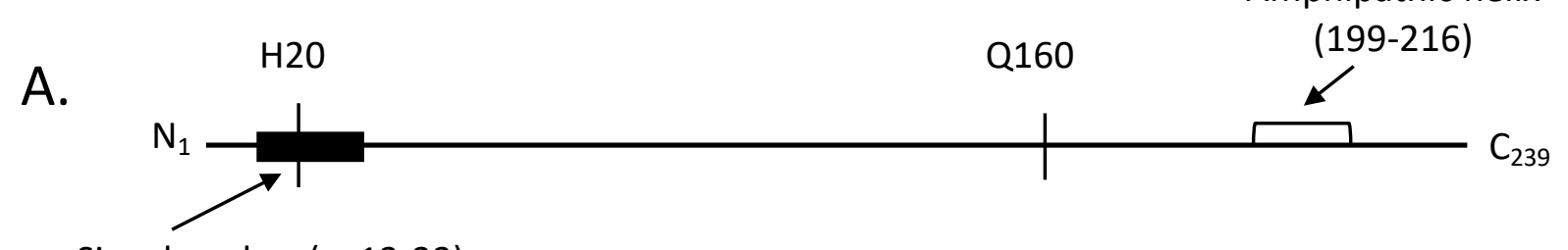

Signal-anchor (aa12-32)

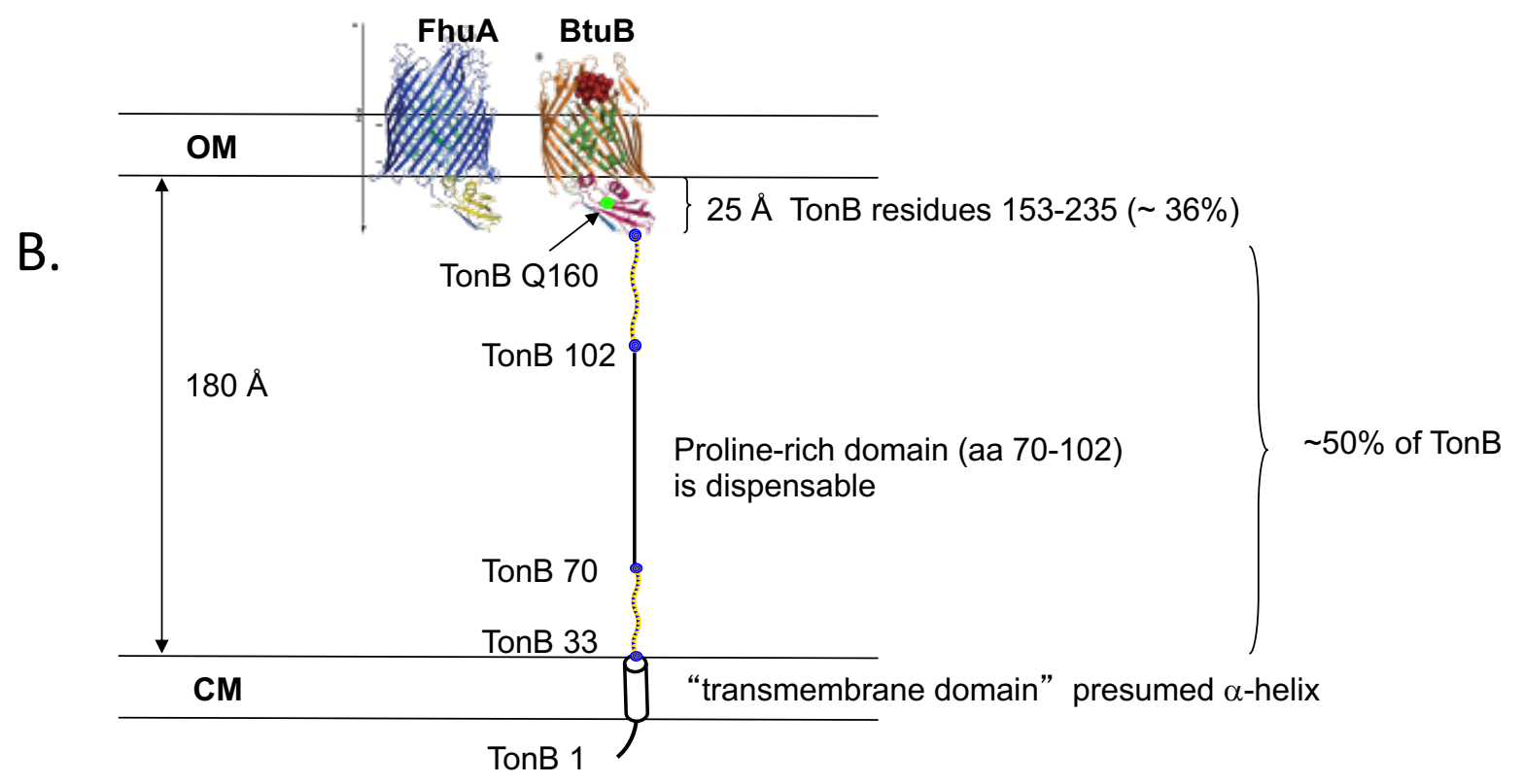

1214 Figure 4. TonB protein information. (A) Relevant features of the TonB

1215 primary amino acid sequence (residues 1-239) are shown. H20 in the TonB

1216 transmembrane signal anchor domain renders TonB inactive when substituted with

1217 alanine (44). Locations of TonB Q160 and the amphipathic helix are shown. (B)

1218 The conformation of TonB predicted by the crystal structures of its carboxy

1219 terminus would be unable to reach the TBDTs. TonB residues 33-69 and 103-149,

1220 predicted to be intrinsically disordered regions (44) are depicted as yellow 
1221 rectangles. TonB Q160 is the green dot within the structured carboxy terminus of

1222 the BtuB-TonB structure. The proline-rich domain, which contributes $\sim 100 \AA$ to

1223 the extension of TonB across the periplasmic space (38), can be deleted without

1224 inactivating TonB $(24,39)$. The span of the periplasmic space was estimated based

1225 on crystal structure reconstructions of the AcrA/B/TolC complex which has

1226 proteins in both outer and cytoplasmic membranes (77). The crystal structure of

1227 FhuA-TonB is from Pawelek et al. (20). The crystal structure of BtuB-TonB is

1228 from Shultis et al. (45). Part B of this figure is from (44). Abbreviations: CM,

1229 cytoplasmic membrane; OM, outer membrane.

1230

1231 

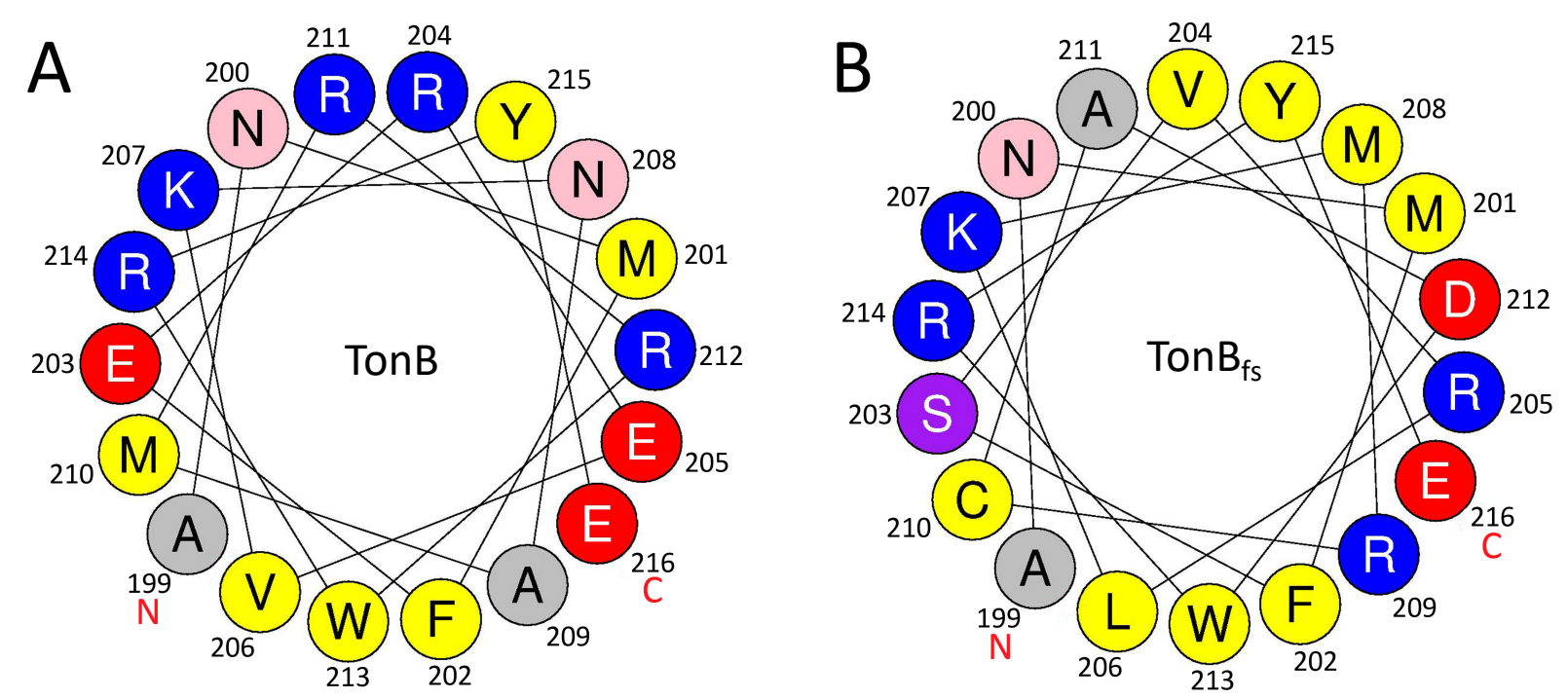
$199\left\{\begin{array}{l}\text { ANMFEREVKNAMRRWRYE } \\ \text { ANMFSVRLKMRCADWRYE }\end{array}\right\} 216$

$$
\text { jnetpred }
$$

JNETCONF

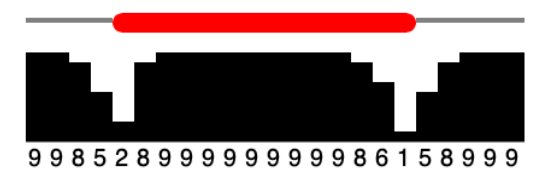

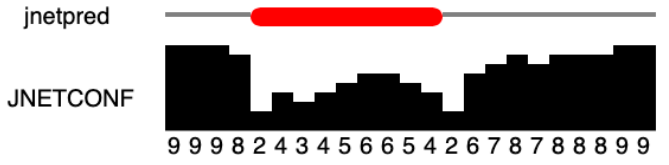

1237 compared to $(\mathbf{B})$ the corresponding frame-shifted region of TonB presented in a

1238 helical wheel diagram with its corresponding JPRED4 prediction (bottom). For

1239 JNetPred, predicted helices are shown in red. For JNETCONF, high values along

1240 the bottom edge indicate high confidence in the prediction (78). The comparison of

1241 the two amino acid sequences is shown in the middle panel, with the frame-shifted

1242 residues noted in red. The frameshifted version has lost much but not all of its 
bioRxiv preprint doi: https://doi.org/10.1101/2021.12.08.471779; this version posted December 8, 2021. The copyright holder for this preprint (which was not certified by peer review) is the author/funder. All rights reserved. No reuse allowed without permission.

1243 helical character and it resulted in multiple substitutions in the core helix residues

$1244 \quad 203-212$.

1245

1246 


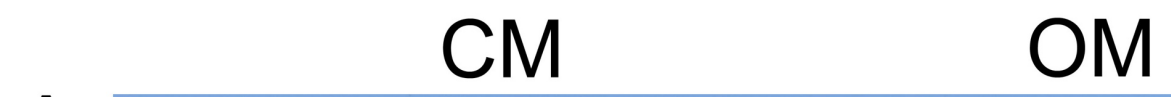

1249

\section{A}

1250

1251

1252

1253

1254

1255

1256

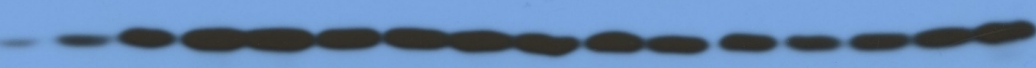

$\varangle$ Monomer TonB $_{\triangle \mathrm{AH}}$

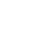

B

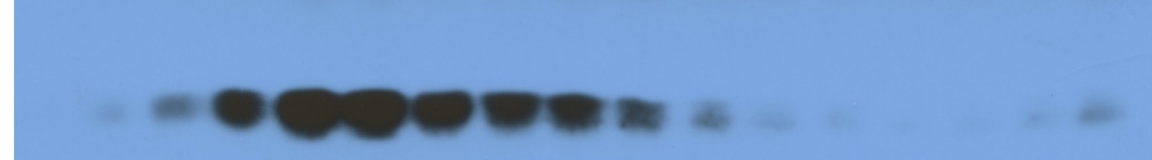

*deg product

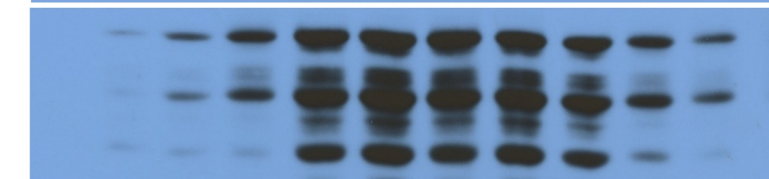

\section{Disulfide- crosslinked complexes}

\section{$\varangle$ Monomer TonB $_{\mathrm{fs}}$}

*deg product 
1267 amount of monomer is apparent in OM fractions on this exposure. On longer

1268 exposures, monomer $\mathrm{TonB}_{\mathrm{fs}}$ association with the outer membrane becomes more

1269 apparent (data not shown). CM is for cytoplasmic membrane fractions; OM is for

1270 outer membrane fractions. Immunoblots of SDS polyacrylamide gels with anti-

1271 TonB monoclonal antibody are shown (71).

1272

1273 
A.
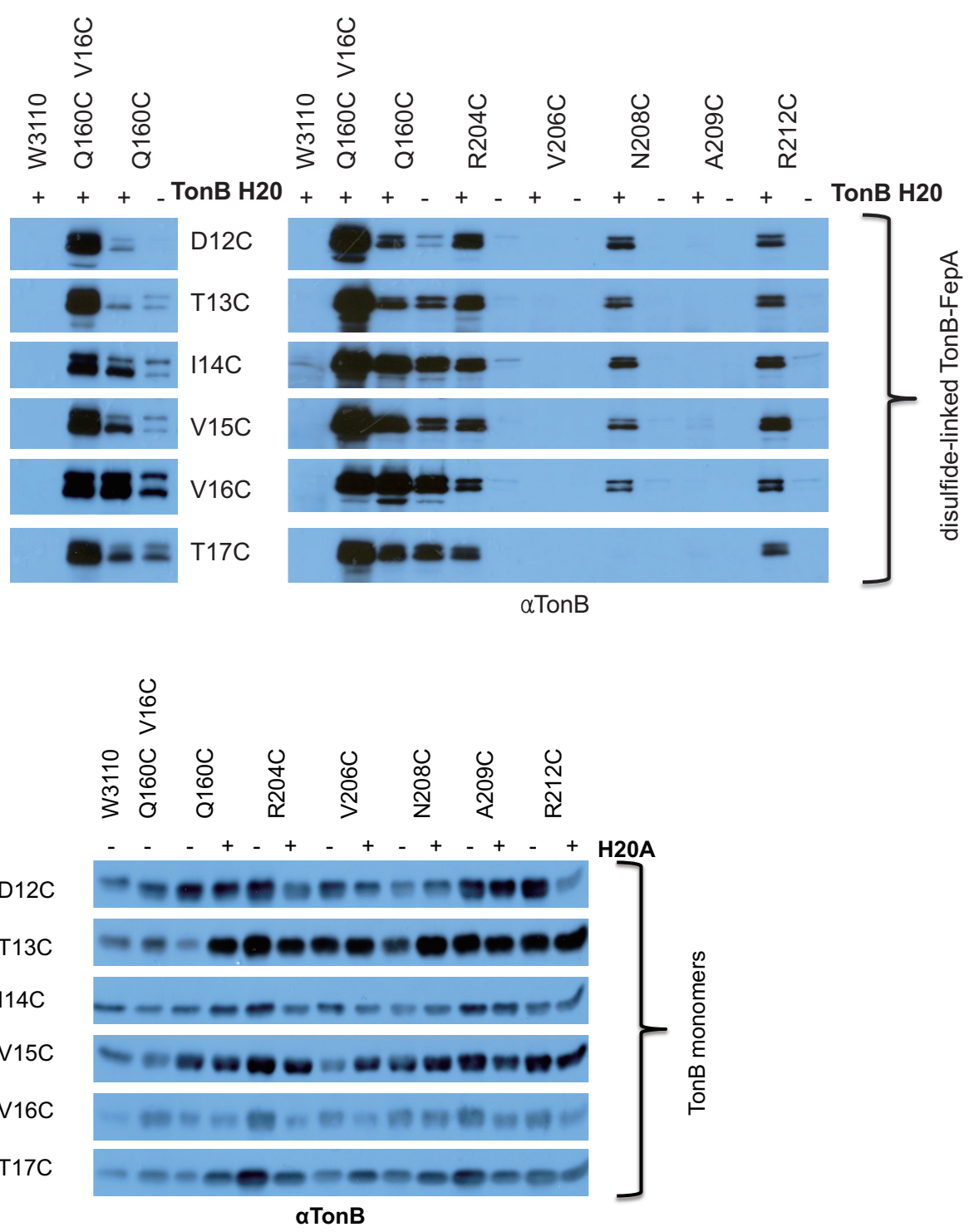

1275 Fig. 7. Cys substitutions in the essential TonB amphipathic helix form in vivo

1276 disulfide crosslinks with FepA TonB box Cys substitutions. Strain KP1491

$1277[\mathrm{~W} 3110 \Delta$ fep $A, \Delta(\operatorname{ton} B, P 14):: k a n]$ with various TonB and FepA plasmid

1278 combinations was grown and analyzed as described in Materials and Methods.

1279 TonB and its Cys substitutions are indicated across the top of the immunoblots. 
1280 FepA Cys substitutions in the TonB box are indicated between the two panels (in

1281 (A) or on the left side of the immunoblot in (B). $(+)$ indicates the presence of the 1282 wild-type $\mathrm{H} 20$ allele in the TonB transmembrane domain. (-) indicates the 1283 presence of the inactivating $\mathrm{H} 20 \mathrm{~A}$ mutation. Immunoblots of the $\sim 116 \mathrm{kDa}$ region 1284 of non-reducing SDS polyacrylamide gels developed with monoclonal anti-TonB 1285 antibody are shown. (A) Wild-type control W3110 shows that wildtype TonB and 1286 wildtype FepA do not innately form stable complexes. Panel right: TonB-FepA 1287 disulfide-linked complexes are shown. Panel left: shorter exposures of the first 1288 four lanes of panel right are shown. In panel left, the TonB Q160C-FepA V16C 1289 pair demonstrated the most efficient crosslinking among five TonB FepA TonB 1290 box Cys substitutions tested and was therefore used as a standard for relative levels 1291 in all subsequent figures characterizing disulfide crosslinks. (B) Steady state levels 1292 of chromosomally encoded TonB in W3110 and plasmid-encoded TonB variants

1293 from samples in (A) are shown as immunoblots of the $\sim 36 \mathrm{kDa}$ region of reducing

1294 SDS polyacrylamide gels developed with anti-TonB monoclonal antibody. 
A

1298

1299

1300

1301

1302

1303

1304

1305

1306

1307

1308

1309

1310

1311

1312

1313

1314

1315

1316

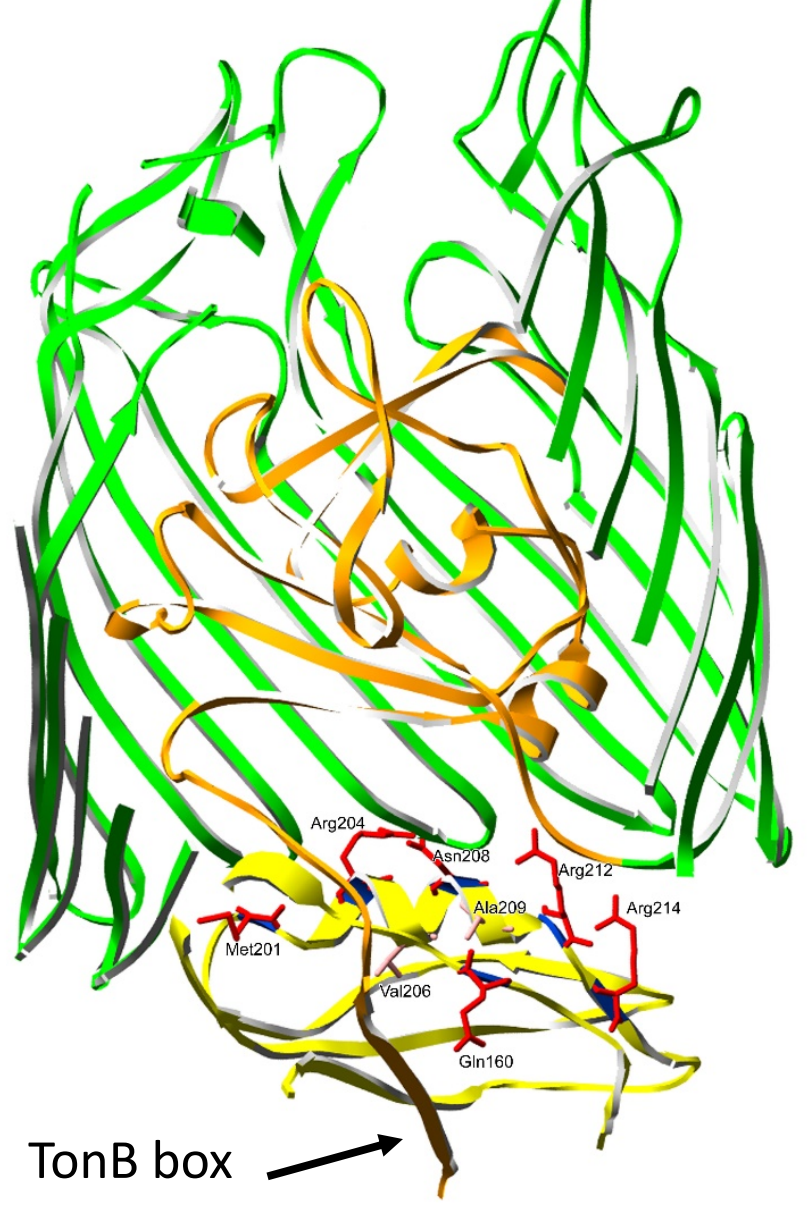

B

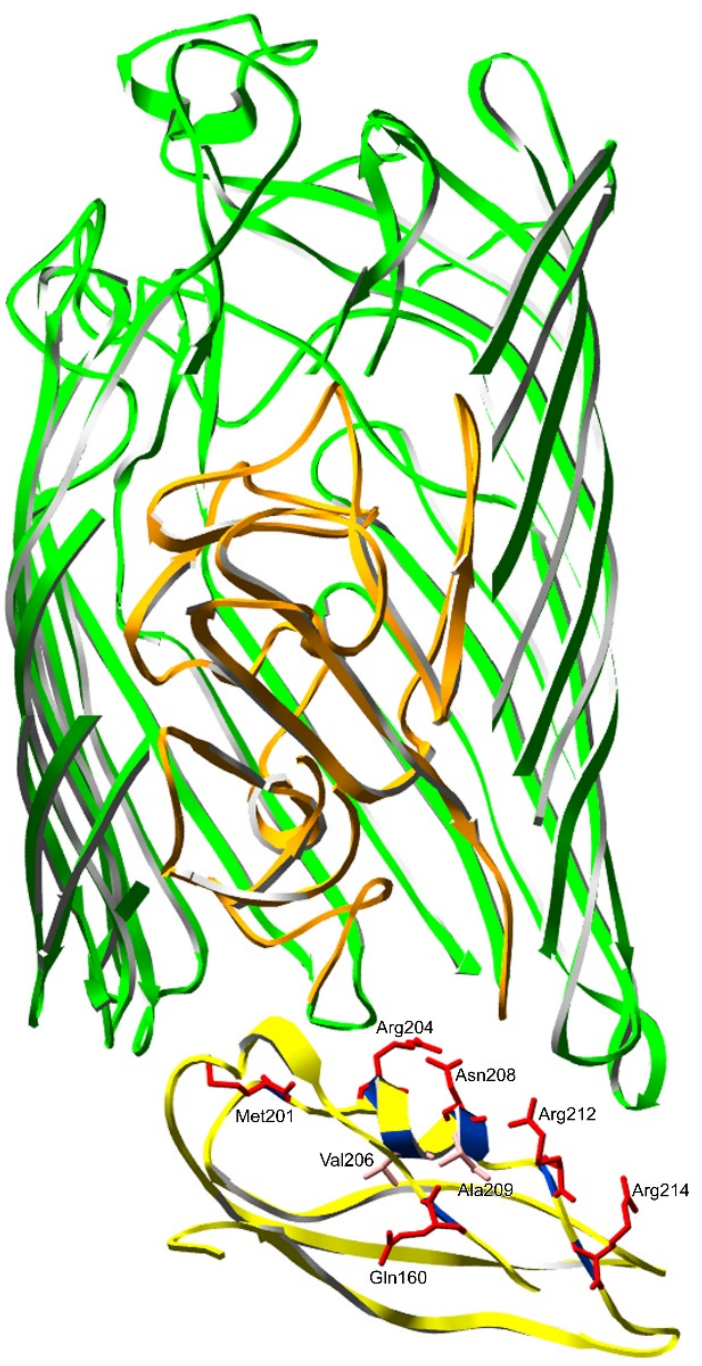

Figure 8: Ribbon diagrams of TonB Cys-substituted residues displayed on

TonB carboxy terminus co-crystal structures with BtuB [A; Shultis et al., (45)]

and FhuA [B; Pawelek et al., (20)]. TonB Cys substitutions from this study that

made disulfide crosslinks with a variety of Cys substitutions in FepA, including the

TonB box, are in red; those in pink (A206C and A209C) made no crosslinks. The

TonB box of BtuB is shown. The FhuA TonB box was not visible in the FhuA- 
1317 TonB structure. The authors of that study propose that FhuA TonB box residues

1318 I9, T10, V11, and A13 interact with TonB residues V225, V226, L229, and K231

1319 on one side and with TonB Q160 on the other (20).

1320

1321

1322

1323

1324

1325

1326

1327

1328

1329

1330

1331

1332

1333

1334

1335

1336 
A

A

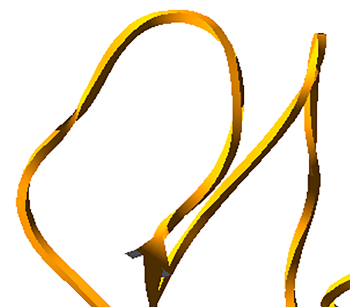

B

1342

1343

1344

1345

1346

1347

1348

1349

1350

1351

1352

1353

Figure 9: The FepA cork, residues 1-85. (A) Ribbon diagram of the FepA cork

1354 showing residues 1-85 in the mechanically weak segment of the FepA cork that were chosen for Cys substitution and in vivo disulfide crosslinking. The external surface of the cork is at the top, the periplasmic surface is at the bottom. The TonB box is indicated. Red residues formed crosslinks; pink residues did not. (B) Space

1358 filling model of the periplasmic surface of FepA, showing periplasmic accessibility 
1359 of the residues labelled in (A). The FepA barrel is light blue; the FepA cork is

1360 light green; the periplasmically accessible residues tested are shown in pink. T51 is

1361 partially accessible; Gly54 and Ala42 (dark blue) are barely visible; Ser46 is

1362 completely buried. The crystal structure was solved by Buchanan et al. (60). 

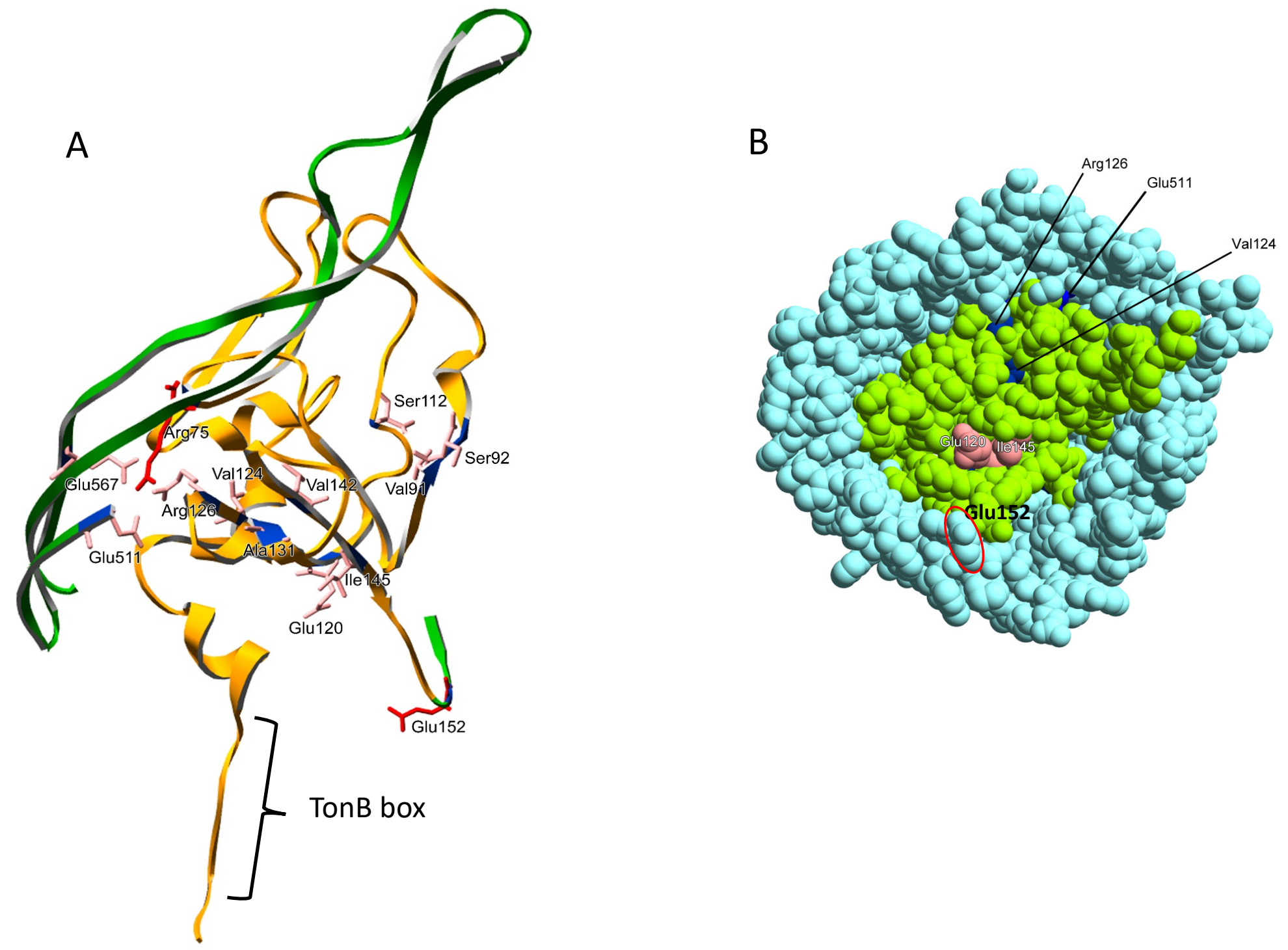

1383 Figure 10. The FepA cork, residues 75-152. (A) Ribbon diagram of the FepA

1384 cork showing residues 75-152, a mechanically recalcitrant segment of the cork,

1385 that were chosen for Cys substitution and in vivo disulfide crosslinking. The

1386 external surface of the cork is at the top, the periplasmic surface is at the bottom.

1387 The TonB box is indicated. Red residues formed crosslinks; pink residues did not. 
1388 Arg75 should be pink instead of red. Portions of the FepA barrel (dark green) show 1389 residues Glu511 and Glu567 which together with cork residues Arg75 and Arg126

1390 form the lock region (54). (B) Space filling model of the periplasmic surface of

1391 FepA, showing periplasmic accessibility of the residues labelled in (A). The FepA

1392 barrel is blue; the FepA cork is light green; periplasmically accessible residues are

1393 shown in pink. Semi-accessible residues are dark blue. Glu 152 is circled in red.

1394 The crystal structure was solved by Buchanan et al. (60).

1395 


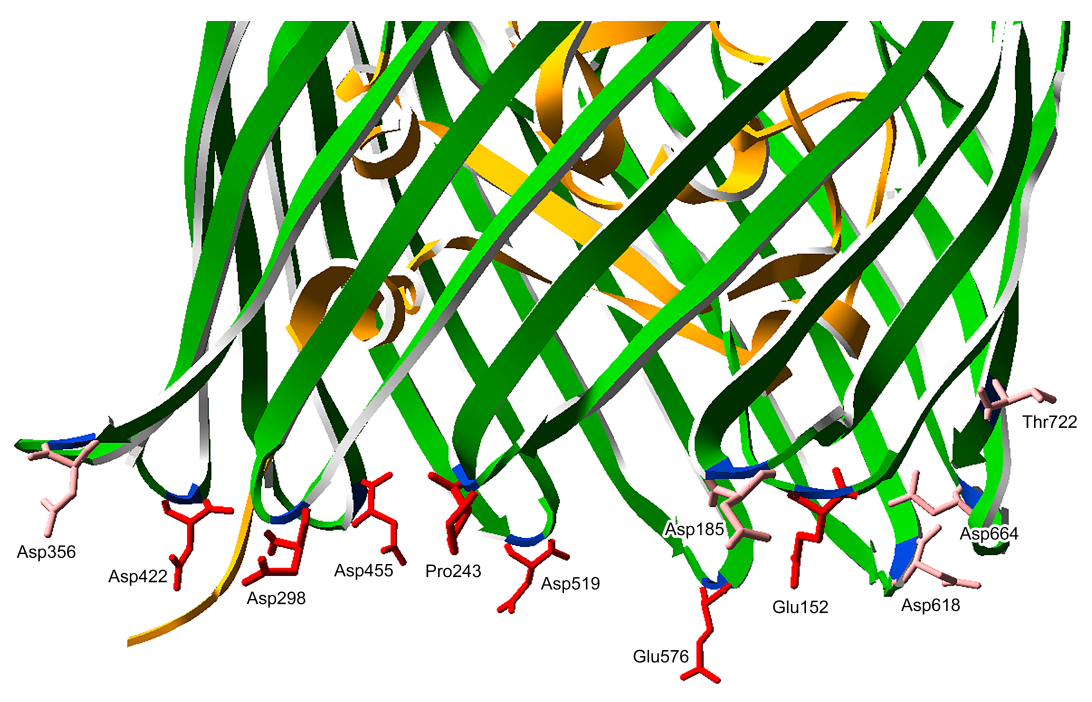

Figure 11. The FepA barrel $\beta$-strand turns. Ribbon diagram of the FepA $\beta$ residues did not. The crystal structure was solved by Buchanan et al. (60). 
A.

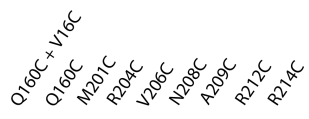

$\mathrm{L} 23 \mathrm{C}$

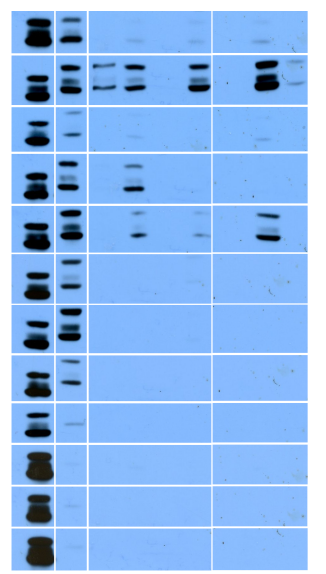

B.

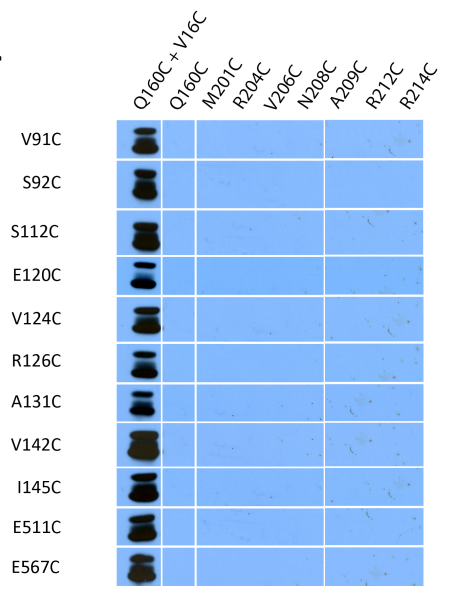

C.

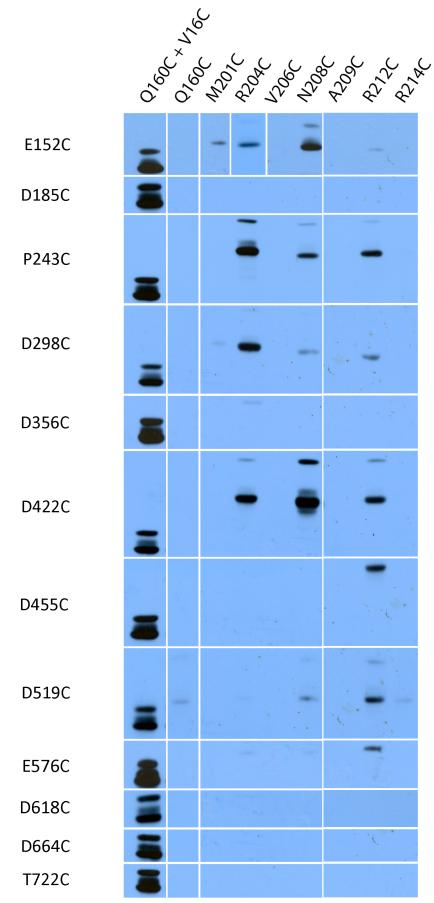

Fig. 12 The composite comparison of in vivo TonB-FepA disulfide

1413 from the mechanically weak region of the FepA cork (residues 1-70) as well as

R75C and L85C from the transition between mechanically weak and mechanically recalcitrant domains. R75 is considered a part of the "lock region". (B) TonB Cys

1417 region of the FepA cork (residues 91-145) and the FepA "lock region" (residues 
1420 transition between the FepA cork and barrel. TonB Cys substitutions are indicated

1421 across the top of the immunoblots. FepA Cys substitutions are indicated along the

1422 left side of each composite. TonB-FepA disulfide-crosslinked complexes were

1423 visualized in strain KP1491[W3110 $\Delta$ fepA, $\Delta(\operatorname{tonB}, P 14): \because k a n]$. Composite

1424 immunoblots of non-reducing SDS polyacrylamide gels with anti-TonB

1425 monoclonal antibody are shown. Since not all experiments were performed on the

1426 same immunoblot, exposures for this composite summary were chosen based on

1427 matching the Q160C + V16C standards among immunoblots (left-most lanes in A, 1428 $\mathrm{B}$, and $\mathrm{C})$. 
A.

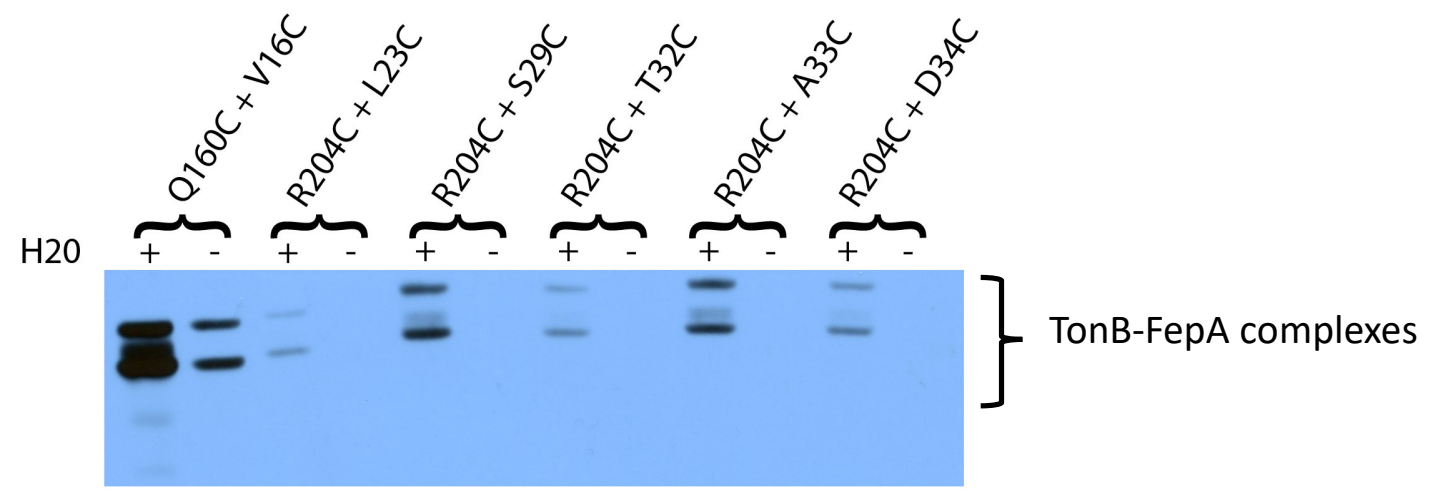

B.

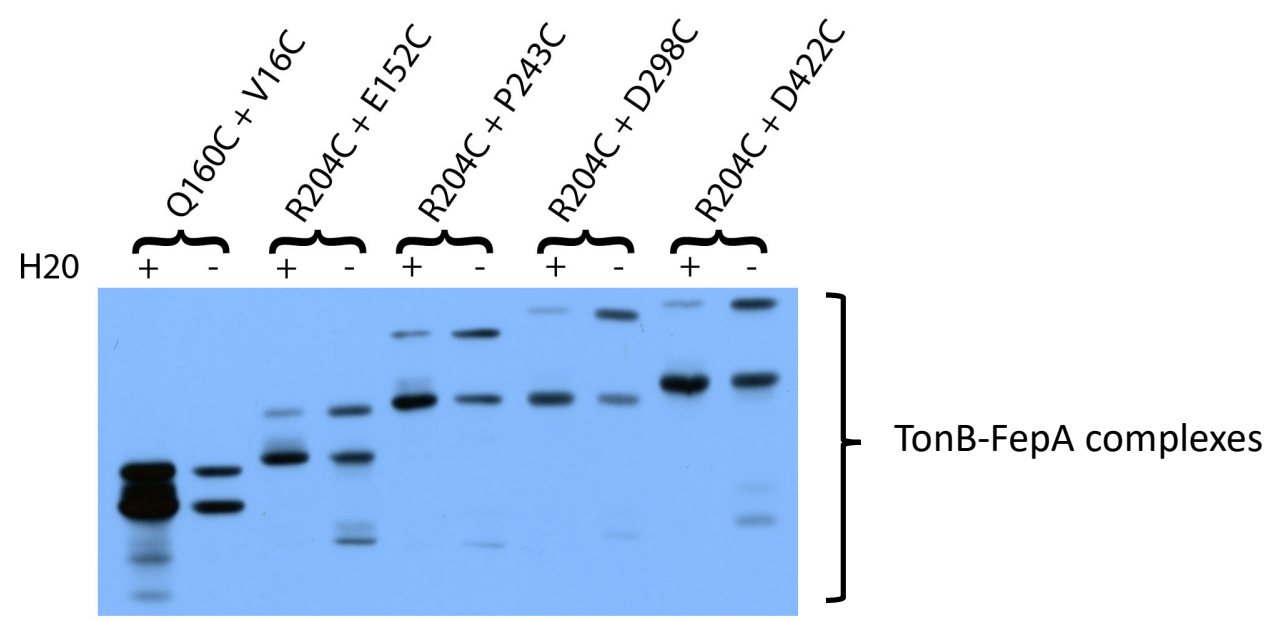

Figure 13: In vivo, TonB R204C makes both functionally important and functionally unimportant disulfide crosslinks with FepA Cys substitutions. top of each set of lanes. H20 (+) indicates the presence of the wild-type H20 allele

1437 in the TonB transmembrane domain. (-) indicates the presence of the inactivating 1438 H20A mutation. (A) The presence of the TonB H20A mutation significantly 1439 reduced disulfide crosslinking by FepA early cork Cys substitutions (depicted in 1440 Fig. 9). B). The H20A mutation has little effect on the overall abundance of 
1441 R204C-mediated disulfide crosslinks at the cork-barrel interface, specifically FepA

1442 E152C (depicted in Fig. 10) or the barrel turns, where they occurred (depicted in

1443 Fig. 11). The TonB Q160C-FepA V16C pair was used as a standard for

1444 comparison of relative levels (far left lanes in A. and B.). TonB-FepA disulfide-

1445 crosslinked complexes were visualized in strain KP1491[W3110 $\Delta$ fepA,

$1446 \Delta(\operatorname{ton} B, P 14):: k a n]$. Immunoblots of non-reducing SDS polyacrylamide gels with

1447 anti-TonB monoclonal antibody are shown. Monomer TonB levels for all samples

1448 in these immunoblots were at or near chromosomal levels (data not shown). 
A.

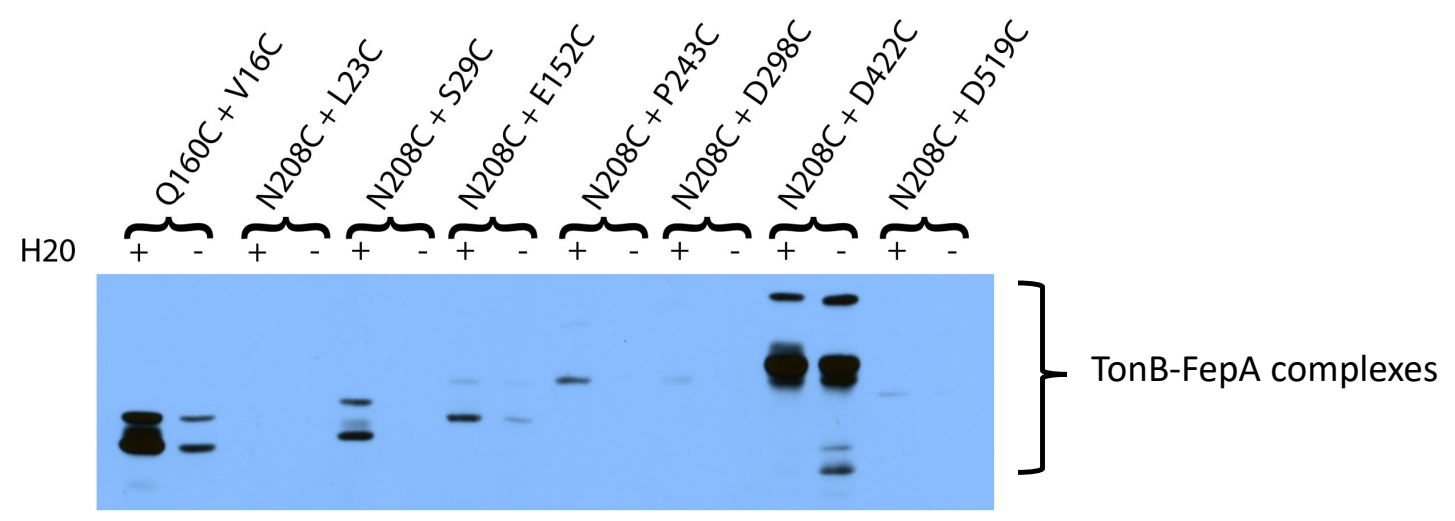

B.

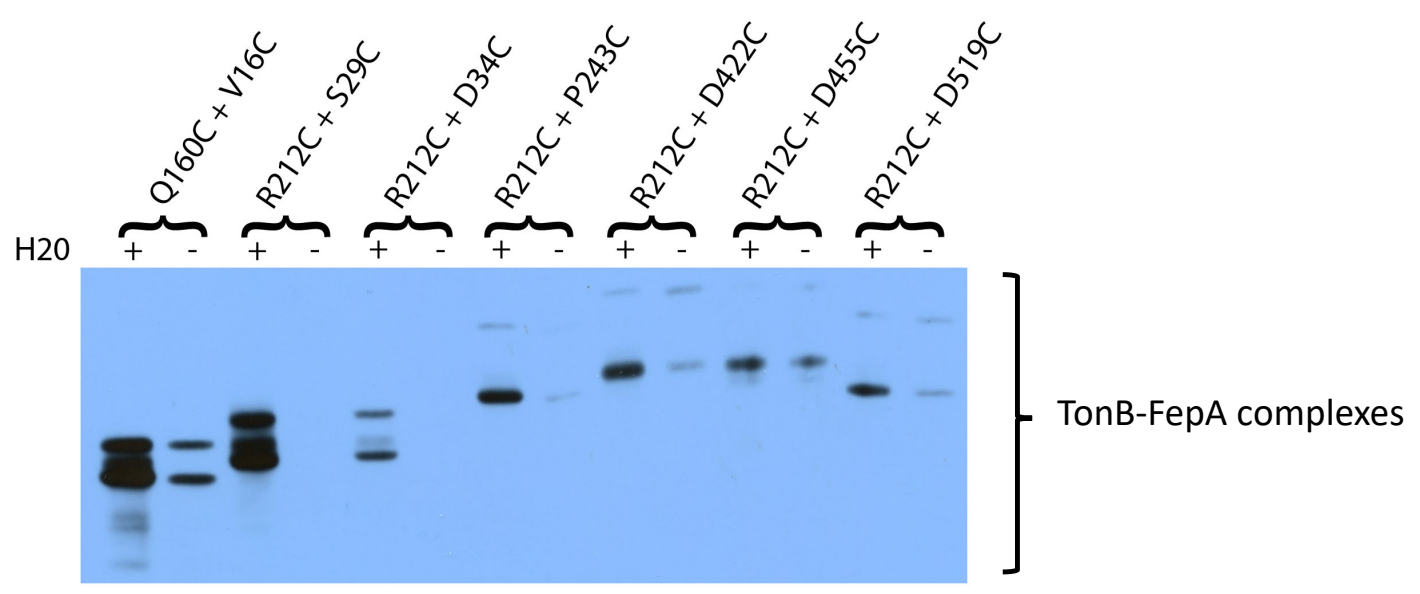

Figure 14: In vivo, TonB N208C and TonB R212C make both functionally 
1461 (depicted in Fig. 11). This interaction was impervious to the presence of the H20A

1462 mutation. (B) Most notably, TonB R212C makes a very abundant, H20-specific,

1463 crosslink with FepA S29C (depicted in Fig. 9). It also makes H20-specific

1464 complexes with some of the FepA Cys substitutions in the barrel turns (depicted in

1465 Fig. 11). The TonB Q160C-FepA V16C pair was used as a standard (far left lanes

1466 in A. and B.). TonB-FepA disulfide-crosslinked complexes were visualized in

1467 strain KP1491[W3110 $\Delta f e p A, \Delta(\operatorname{ton} B, P 14): \because k a n]$. Immunoblots of non-reducing

1468 SDS polyacrylamide gels with anti-TonB monoclonal antibody are shown.

1469 Monomer TonB levels for all samples in these immunoblots were at or near

1470 chromosomal levels (data not shown). 
A.

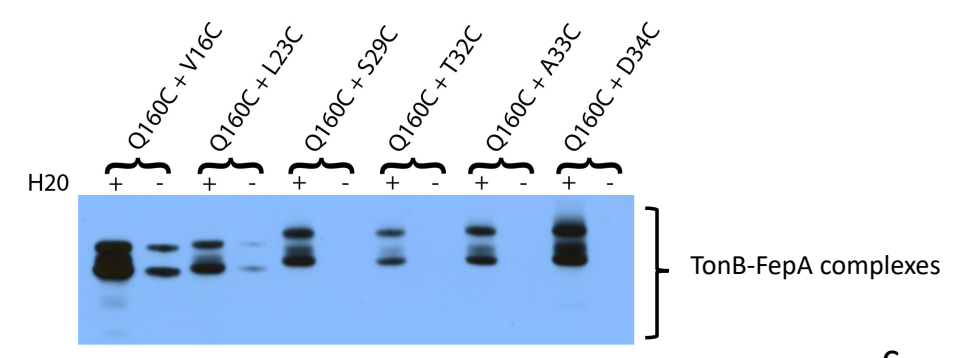

C.

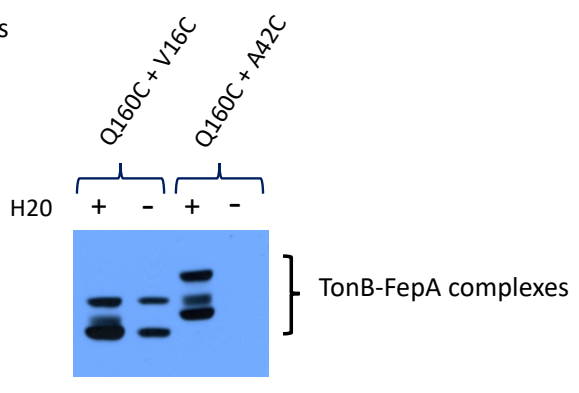

B.

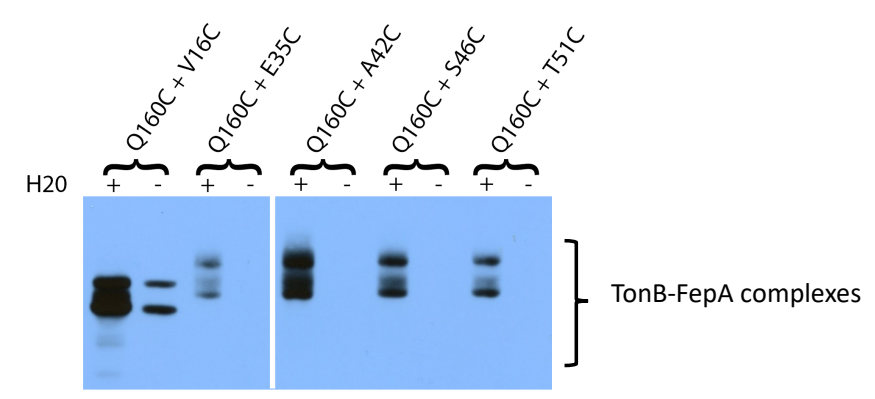

Figure 15: In vivo, TonB Q160C makes several functionally important

disulfide crosslinks within the mechanically weak segment of the FepA cork,

FepA Cys substitution combinations are indicated at the top of each lane. H20 (+) 
1488 entirely prevented by the presence of the TonB H20A mutation. For this composite

1489 immunoblot, the exposure on the right was chosen based on matching it to the

1490 same intensity as the Q160 + V16C standard shown in the left panel. (C) Direct

1491 comparison of Q160C complexes with FepA V16C and FepA A42C on the same

1492 immunoblot and with a shorter exposure. TonB-FepA disulfide-crosslinked

1493 complexes were visualized in strain KP1491[W3110 $\Delta$ fepA, $\Delta($ tonB,P14)::kan].

1494 Immunoblots of non-reducing SDS polyacrylamide gels with anti-TonB

1495 monoclonal antibody are shown. Monomer TonB levels for all samples in these

1496 immunoblots were at or near chromosomal levels (data not shown). 

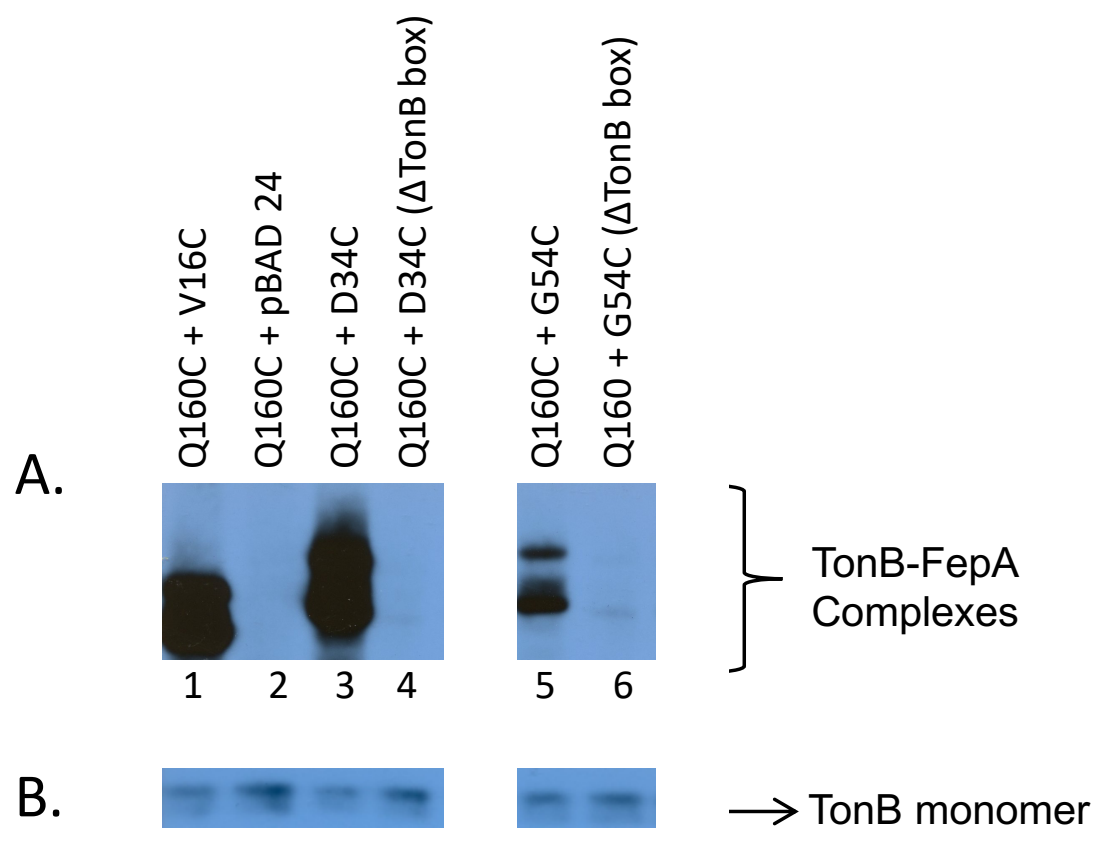

$\alpha$ TonB

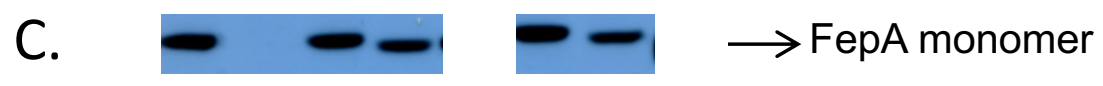

aFepA

1502 formation by TonB Q160C. (A) TonB-FepA disulfide-crosslinked complexes

1503 visualized in strain KP1491[W3110 $\Delta$ fepA, $\Delta($ tonB,P14) $:: k a n]$ by immunoblots of

1504 non-reducing SDS polyacrylamide gels with anti-TonB monoclonal antibody.

1505 pBAD24 is the vector into which fepA variants were cloned. (B) Monomer levels

1506 of TonB from the same samples as A), visualized by anti-TonB monoclonal

1507 antibody. (C) Monomer levels of FepA from same samples as A.), visualized by 
bioRxiv preprint doi: https://doi.org/10.1101/2021.12.08.471779; this version posted December 8, 2021. The copyright holder for this preprint (which was not certified by peer review) is the author/funder. All rights reserved. No reuse allowed without permission.

1508 anti-FepA polyclonal antibody. All lanes are from the same immunoblot with a 1509 center lane masked. 


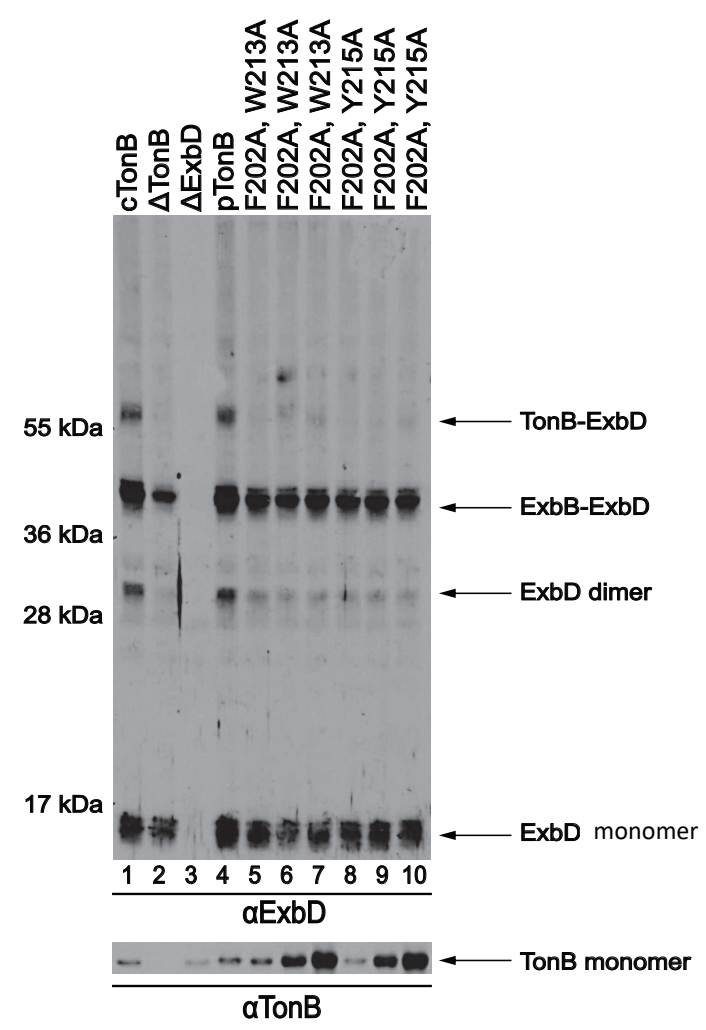


1523 samples above are shown. For pKP531 and pKP532, note the increase in TonB

1524 expression with increasing addition of the inducer, arabinose.

1525

1526 


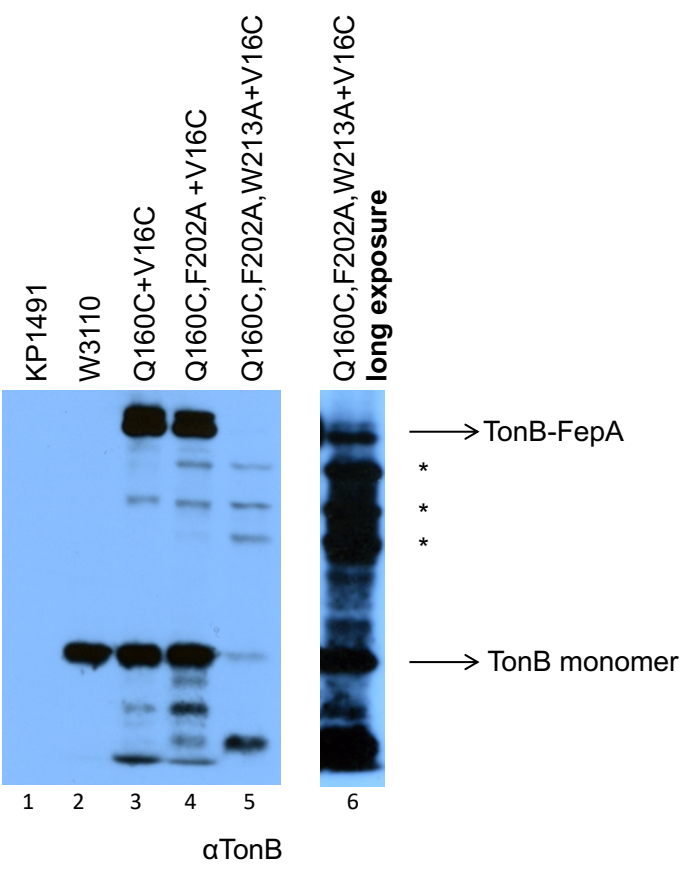

Figure 18. Inactivation of the TonB carboxy terminus appears to divert TonB 
1540 SDS polyacrylamide gels developed with monoclonal anti-TonB antibody are

1541 shown.

1542

1543

1544

1545

1546

1547 